\title{
The Critical Role of Non-Normality in Partitioning Tropical and Extratropical Contributions to PNA Growth
}

\author{
StePhanie A. Henderson AND DANiEl J. Vimont \\ Department of Atmospheric and Oceanic Sciences, University of Wisconsin-Madison, Madison, Wisconsin \\ MATTHEW NEWMAN \\ Cooperative Institute for Research in Environmental Sciences, University of Colorado Boulder, and Physical Sciences \\ Laboratory, NOAA/Earth System Research Laboratories, Boulder, Colorado
}

(Manuscript received 22 July 2019, in final form 27 April 2020)

\begin{abstract}
The Pacific-North American (PNA) teleconnection pattern has been linked both to tropical phenomena, including the Madden-Julian oscillation (MJO) and El Niño-Southern Oscillation (ENSO), and to internal extratropical processes, including interactions with the zonally varying basic state and synoptic eddies. Many questions remain, however, concerning how these various relationships act, both separately and together, to yield observed PNA variability. Using linear inverse modeling (LIM), this study finds that the development and amplification of PNA anomalies largely results from the interference of modes strongly coupled to sea surface temperatures (SST), such as ENSO, and modes internal to the atmosphere, including the MJO. These SST-coupled and "internal atmospheric" modes form subspaces that are not orthogonal, and PNA growth is shown to occur via non-normal interactions. An internal atmospheric space LIM is developed to examine growth beyond this interference by removing the SST-coupled modes, effectively removing ENSO and retaining MJO variability. Optimal PNA growth in the internal atmospheric space LIM is driven by MJO heating, particularly over the Indian Ocean, and a retrograding northeast Pacific streamfunction anomaly. Additionally, the individual contributions of tropical heating and the extratropical circulation on PNA growth are investigated. The non-normal PNA growth is an important result, demonstrating the difficulty in partitioning PNA variance into contributions from different phenomena. This cautionary result is likely applicable to many geophysical phenomena and should be considered in attribution studies.
\end{abstract}

\section{Introduction}

The Pacific-North American (PNA) pattern is a dominant pattern of boreal winter Northern Hemisphere lowfrequency variability, with significant impacts on North American temperature and precipitation anomalies (e.g., Dickson and Namias 1976; Leathers et al. 1991; Franzke et al. 2011). First identified by Wallace and Gutzler (1981), the PNA is characterized by a wavelike anomaly pattern over the North Pacific and North America, which robustly emerges using various statistical techniques such as rotated empirical orthogonal function (EOF) analysis, $k$-means clustering, and one-point correlation maps (e.g., Straus and Shukla 2002; Casola and Wallace 2007; Mo and Ghil 1988).

Corresponding author: Stephanie A. Henderson, sahenderson@ wisc.edu
Studies have found that both tropical heating and extratropical synoptic eddy dynamics impact PNA pattern development and growth. Mori and Watanabe (2008) demonstrated that tropical heating associated with the Madden-Julian oscillation (MJO; Madden and Julian 1994; Zhang 2005), the leading form of intraseasonal variability in the tropics, can precede and trigger a PNA pattern. Franzke et al. (2011) found that similar convective anomalies force an initial PNA pattern. Riddle et al. (2012) used $k$-means cluster analysis to show that positive and negative PNA pattern clusters were more likely to occur following certain MJO phases. The teleconnection pattern response to El NiñoSouthern Oscillation (ENSO) heating has also been linked to the PNA (e.g., Horel and Wallace 1981; Hoerling et al. 1997; Johnson and Feldstein 2010), while other studies argue that the PNA pattern and ENSO teleconnections are distinct (e.g., Straus and Shukla 
2002, 2000), and that ENSO instead has a stronger projection onto the tropical/Northern Hemisphere pattern (TNH; e.g., Livezey and Mo 1987).

While tropical convection like the MJO can trigger an initial PNA pattern, PNA amplification is thought to be largely driven by extratropical eddy dynamics via barotropic amplification through interactions with the climatological zonally varying flow and eddy vorticity advection (e.g., Simmons et al. 1983; Branstator 1990, 1992; Borges and Hartmann 1992; Borges and Sardeshmukh 1995; Mori and Watanabe 2008; Franzke et al. 2011). Although PNA structures are typically preceded by tropical convection (e.g., Dai et al. 2017), this is not always the case. Johnson and Feldstein (2010) found that certain PNA-like patterns are weakly related to tropical convection. Furthermore, Dai et al. (2017) demonstrated that some PNA events are preceded by weak or less organized tropical convection. In such cases, extratropical dynamics must play a larger role.

The literature strongly suggests that both tropical heating and the extratropical circulation are important for PNA development. However, it is not clear how and how much each contribute to PNA growth, or what are the relative roles within the tropical heating of the MJO and ENSO. For example, it is not clear why only some MJO events trigger a PNA pattern (e.g., Henderson et al. 2016). One possibility is that both the tropical convection and the extratropical environment must be favorable. Another possibility is that only certain MJO phases are important for PNA pattern development (e.g., Newman and Sardeshmukh 1998; Henderson et al. 2016; Mori and Watanabe 2008). The role of ENSO is even less clear, given the discrepancy in previous studies. Overall, while studies have tended to focus on the roles of the MJO and ENSO on the PNA pattern independently, what seems desirable is for a comprehensive analysis of PNA development to consider all relevant processes within the same dynamical system.

In this paper, we argue that non-normality, a fundamental characteristic of linear dynamical systems with asymmetric interactions between state variables, is crucial for understanding and diagnosing observed PNA evolution. For example, many climate processes have a strong dependence upon location (e.g., advection in a sheared flow; Farrell 1982; Boyd 1983). There are also unequal dependencies between independent system variables (e.g., surface atmospheric winds drive surface ocean currents but not vice versa; Moore and Kleeman 1999). These asymmetries mean that the climate system's dynamical operator is asymmetric (i.e., not self-adjoint), so its eigenmodes are nonorthogonal (e.g., Strang 2006). It follows that anomaly growth over finite periods of time may occur, even in the absence of exponential instability, due to similarly structured but differently evolving eigenmodes progressing from destructive to constructive interference (e.g., Farrell 1988; Farrell and Ioannou 1996). For any prespecified metric, "optimal" initial conditions can therefore be found that maximize anomaly growth (e.g., Borges and Hartmann 1992; Penland and Sardeshmukh 1995; Penland and Matrosova 2006; Sardeshmukh et al. 1997; Winkler et al. 2001) and predictability (Newman et al. 2003) over any time interval.

Consequently, in this paper we examine optimal PNA growth within a linear, non-normal dynamical system that captures observed wintertime coupled tropicalextratropical interactions in the Northern Hemisphere. This dynamical system is determined empirically using linear inverse modeling (LIM), in which coarse-grained nonlinear system dynamics are approximated as the sum of slowly evolving (predictable) linear dynamics and rapidly evolving (unpredictable) noise, both determined from the observed covariability statistics of the system. The goal of this analysis is to produce a dynamical model in an inverse sense (i.e., from system output) that has a form similar to what could be produced in a forward sense from an appropriate analysis of the physical dynamical equations.

The LIM's ability to produce forecast skill competitive with operational models directly supports its relevance to predictability studies and process-based diagnosis of lowfrequency variability. For example, Albers and Newman (2019) showed that skill of their wintertime LIM for forecast leads of 3-6 weeks was comparable to the operational coupled models currently in use at both NCEP and ECMWF. Studies have employed LIM to diagnose and investigate the predictability of the extratropical circulation (e.g., Winkler et al. 2001; Newman and Sardeshmukh 2008) and sea surface temperatures (SST; e.g., Penland and Sardeshmukh 1995; Penland and Matrosova 1998; Newman 2007, 2013), as well as specific climate patterns including the Atlantic meridional mode (AMM; e.g., Vimont 2012), the Pacific decadal oscillation (PDO; Newman et al. 2016; Alexander et al. 2008), eastern and central Pacific ENSO events (e.g., Vimont et al. 2014; Penland and Sardeshmukh 1995), the PNA (Cash and Lee 2001), and, recently, North Pacific blocking (Breeden et al. 2020).

Our primary aim is to determine if optimal PNA growth can be characterized as non-normal and, if so, how much of it arises from the constructive interference of MJO and ENSO anomalies. This interference is investigated through the development of a LIM that includes both ENSO and MJO in the state vector through the inclusion of reanalysis-based unfiltered tropical heating. We distinguish the separate roles of MJO and ENSO by identifying the subspace (i.e., the eigenmodes) of the dynamical operator with strong SST coupling, which includes ENSO, and the subspace of the variability internal to the 

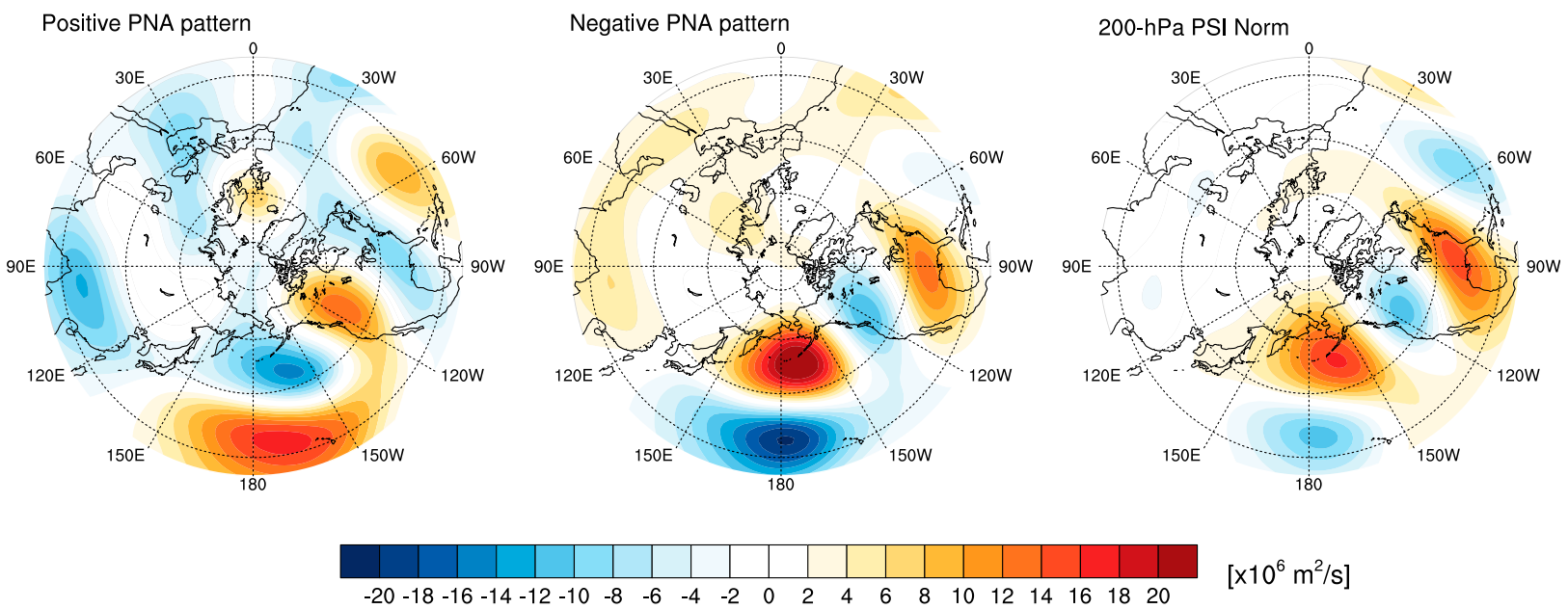

FIG. 1. Composites of 200-hPa streamfunction anomalies for (left) positive and (center) negative PNA days, defined when the CPC PNA index (used in the calculation of the PNA norm) exceeds 1.5 standard deviations. Also shown is (right) the PNA norm projected onto the full LIM 200-hPa streamfunction EOFs (arbitrarily scaled to have a comparable magnitude to the PNA pattern composites).

atmosphere, which includes the MJO (e.g., Newman et al. 2009). An "internal atmospheric" LIM representing the dynamics internal to the atmosphere is developed from the latter to examine optimal PNA growth beyond the interference of the two subspaces. In other words, the internal atmospheric LIM allows for the identification of variability internal to the atmosphere that leads to PNA growth, such as the MJO and midlatitude dynamics, while excluding contributions from ENSO. Although a LIM-based filter has been previously used to isolate ENSO-related variability within the tropics (e.g., Newman et al. 2009), an internal space LIM has not been developed for use in the extratropics prior to this study to the knowledge of the authors.

Section 2 describes the data used and provides a basic background on LIM. The optimal initial conditions leading to PNA growth are discussed in section 3. These optimal conditions are decomposed in section 4 , and the interference between SST-coupled and SST-uncoupled or weakly coupled modes is examined. This result motivates the development of an "internal space" LIM in section 5, derived using a state vector that excludes the strongly SST-coupled modes (including ENSO) from the dynamical operator. The individual contributions of tropical heating and the extratropical circulation on PNA growth are discussed in section 6 for both the "full" LIM, which includes the SST-coupled modes, and the internal space LIM. Last, a summary of the main findings and concluding remarks are provided in section 7 .

\section{Linear inverse modeling}

\section{a. Data}

The LIM is derived using wintertime [DecemberFebruary (DJF)] daily averaged data calculated from the 6-hourly ERA-Interim reanalysis dataset (Dee et al. 2011), which have a $1.5^{\circ} \times 1.5^{\circ}$ resolution. This includes 200 - and 850-hPa streamfunction calculated from the daily zonal and meridional winds, and the apparent heat source (Q1), calculated as a residual from the dry static energy budget following Yanai et al. (1973). The LIM also incorporates DJF SST data, which are obtained from the NOAA Optimum Interpolation Sea Surface Temperature (OISST) dataset (Reynolds et al. 2002) and averaged onto a $2^{\circ}$ latitude $\times 5^{\circ}$ longitude grid. The DJF seasons consist of 90 days each and span from December 1982 to February 2016, for a total of 33 boreal winter seasons. Prior to any analysis, each variable's annual cycle and long-term mean were removed, and a 5-day running mean was applied as a coarse-grained low-pass filter.

The PNA is defined using the daily NOAA-NCEP Climate Prediction Center (CPC) PNA index. The index is based on rotated principal component analysis of $20^{\circ}-$ $90^{\circ} \mathrm{N}$ anomalous 500-hPa geopotential height. For more details on the PNA index calculation see CPC (2017). Positive and negative PNA event representation by this index are shown in Fig. 1. The opposite-sign DJF anomalous $200-\mathrm{hPa}$ streamfunction composites consist of a large-scale quadrupole extending from the subtropical Pacific to southeastern North America. We note that this is a simplification of the PNA as there is no one single pattern that is used to define the PNA (e.g., Johnson and Feldstein 2010). It is beyond the scope of this study to examine a wide range of PNA-like patterns using LIM.

\section{b. Linear inverse modeling}

Given that LIM is described in detail in various papers (e.g., Penland and Sardeshmukh 1995) only a brief 
overview is provided here. LIM uses the observed statistics of a system represented by the coarse-grained state vector $\mathbf{x}$ to determine its dynamical properties and approximate its evolution through a linear stochastic equation:

$$
d \mathbf{x} / d t=\mathbf{L x}+\xi
$$

where the dynamical system matrix $\mathbf{L}$ includes the linearized dynamics of $\mathbf{x}$ as well as the linear approximation to the nonlinear dynamics, and $\xi$ represents stochastic white noise forcing. The deterministic evolution from an initial time 0 to time $\tau$ is the solution to the homogeneous term in (1):

$$
\mathbf{x}(\tau)=\mathbf{G}_{\tau} \mathbf{x}(0)=\exp (\mathbf{L} \tau) \mathbf{x}(0) .
$$

Equation (2) shows that the propagator $\mathbf{G}_{\tau}$ operates on an initial condition $\mathbf{x}(0)$ to yield $\mathbf{x}(\tau)$ at lag $\tau$. The propagator $\mathbf{G}_{\tau}$, and in turn $\mathbf{L}$, is estimated from the simultaneous $\mathbf{C}_{0}$ and lagged $\mathbf{C}_{\tau}$ covariance of $\mathbf{x}$ :

$$
\mathbf{G}_{\tau}=\mathbf{C}_{\tau} \mathbf{C}_{0}^{-1}
$$

and based on (2)

$$
\mathbf{L}=\ln \left(\mathbf{G}_{\tau}\right) / \tau_{0} .
$$

Once $\mathbf{L}$ is determined at a specified lag $\tau_{0}$, a final condition $\mathbf{x}(\tau)$ can be generated at any lag $\tau$ by calculating a new $\mathbf{G}_{\tau}=\exp (\mathbf{L} \tau)$ from (3b). For both the full and internal space LIMs, a value of $\tau_{0}=5$ days is used. This initial lag is found to provide a stable estimate of $\mathbf{L}$ in both LIMs based on the $\tau$ test of Penland and Sardeshmukh (1995) (not shown).

In the case that $\mathbf{x}$ is statistically stationary, all eigenvalues of $\mathbf{L}$ will have negative real parts indicating decay. However, $\mathbf{L}$ is generally non-normal, meaning that its eigenvectors are not orthogonal and hence can project onto one another to create initial structures that can grow over a finite time (Farrell 1988). Growth at lag $\tau$, $\mu(\tau)$, is calculated under a specified norm $\mathbf{N}$ by solving the generalized eigenvalue problem (e.g., Farrell 1988; Martinez-Villalobos and Vimont 2016):

$$
\mathbf{G}_{\tau}^{\mathrm{T}} \mathbf{N} \mathbf{G}_{\tau} \mathbf{p}-\mu(\tau) \mathbf{p}=0,
$$

where $\mathbf{p}$ are the eigenvectors of $\mathbf{G}_{\tau}^{\mathrm{T}} \mathbf{N} \mathbf{G}_{\tau}$ and superscript $\mathrm{T}$ indicates the transpose. The eigenvectors $\mathbf{p}$ are initial structures that experience maximum growth under the norm $\mathbf{N}$, with growth equal to the eigenvalue $\mu(\tau)$. The leading optimal initial structure $\mathbf{p}_{1}$ is then the $\mathbf{p}$ that maximizes growth $\mu(\tau)$, found by the largest eigenvalue $\lambda_{1}$.
Two independent LIMs with different state vectors $\mathbf{x}$ from (1) are diagnosed in this study. The first we refer to as the "full" LIM, which is described in section 3. The second is the "internal atmospheric" LIM, which is based on a second state vector designed to exclude variability strongly coupled to tropical SST, such as ENSO (see section 5). The state vectors for each LIM are defined using a reduced EOF space of tropical and extratropical variables chosen to capture the impact of tropical variability, including the MJO and ENSO, on the PNA pattern as well as the impact of the extratropical circulation on the PNA pattern. These state vectors are described for the full and internal space LIM in sections 3 and 5, respectively.

In this study we are interested in identifying the optimal initial conditions that lead to growth of the PNA pattern. For both the full and internal space LIMs, $\mathbf{N}$ in (4) is calculated from the negative PNA composite pattern shown in Fig. 1 (an oppositesigned norm was also tested using the positive PNA composite pattern, and similar results are found). For each corresponding LIM, the PNA composite pattern is projected onto the full or internal space extratropical state space EOFs (200- and $850-\mathrm{hPa}$ streamfunction), with the regions outside of the PNA tripole (i.e., the northernmost Pacific anomaly and the dipole over North America) set to zero. This projection results in two vectors, $\mathbf{r}_{\Psi_{200}}$ and $\mathbf{r}_{\Psi_{850}}$, which are applied to a norm vector $\mathbf{r}_{\mathrm{PNA}}=\left[\begin{array}{llll}0 & 0 & \cdots & \mathbf{r}_{\Psi_{200}} \\ \mathbf{r}_{\Psi_{550}}\end{array}\right]$, where the tropical state vector variables are set to zero. This is similar to the blocking norm employed by Breeden et al. (2020). The vector $\mathbf{r}_{\text {PNA }}$ is normalized to unit length and $\mathbf{N}$ is calculated as

$$
\mathbf{N}=\mathbf{r}_{\mathrm{PNA}} \mathbf{r}_{\mathrm{PNA}}^{\mathrm{T}}+\epsilon \mathbf{l} .
$$

Following Vimont et al. (2014) and Tziperman et al. (2008), the identity matrix I is multiplied by a small scalar $\epsilon=10^{-9}$ and added in (5) for numerical stability. For reference, the PNA norm projected onto the full LIM 200-hPa streamfunction EOFs is provided in Fig. 1 (right panel).

\section{The full LIM}

We first construct the full LIM from the state vector

$$
\mathbf{x}(t)=\left[\begin{array}{c}
\mathbf{T}_{0} \\
\mathbf{H} \\
\boldsymbol{\Psi}_{200} \\
\boldsymbol{\Psi}_{850}
\end{array}\right],
$$


where we chose variables similar to those used in the tropics-only study of Newman et al. (2009). The state vector is defined within a 52-component reduced EOF space, using the leading principal components (PCs) of tropical $\left(20^{\circ} \mathrm{S}-15^{\circ} \mathrm{N}\right)$ SST anomalies $\mathbf{T}_{0}$, apparent heat source (Q1; Yanai et al. 1973) anomalies $\mathbf{H}$ integrated from the surface up to $200 \mathrm{hPa}$, and extratropical $\left(15^{\circ} \mathrm{N}\right.$ to $\left.90^{\circ} \mathrm{N}\right) 200$ and $850-\mathrm{hPa}$ streamfunction anomalies $\left(\boldsymbol{\Psi}_{200}\right.$ and $\boldsymbol{\Psi}_{850}$, respectively). These variables are comparable to the state vector used in Winkler et al. (2001) to examine tropical-extratropical interactions using a Euclidean, or L2 norm, in (4), in which $\mathbf{N}$ is the identity matrix. However, tropical SST anomalies $\mathbf{T}_{0}$ are included here for the derivation of the internal space LIM (see section 5). We note that a state vector that excludes SST still retains all effects of SST within the atmospheric state. Consequently, a LIM constructed from an atmospheric-only state vector (e.g., Winkler et al. 2001; Cash and Lee 2001) implicitly retains ENSO dynamics [see Fig. 13 from Winkler et al. (2001)] and is therefore different than the internal space LIM derived in this study (see section 5), which explicitly removes strongly SST-coupled dynamical modes like ENSO. Inclusion of SST in the state vector also does lengthen the time scale of development of ENSOrelated diabatic heating and extratropical streamfunction anomalies, resulting in slightly different L2 optimal initial and final conditions than in Winkler et al. (2001) (not shown). The variance retained by the truncated fields is $72 \%, 39 \%, 70 \%$, and $70 \%$, for $\mathbf{T}_{0}, \mathbf{H}, \mathbf{\Psi}_{200}$, and $\boldsymbol{\Psi}_{850}$, respectively, explained by the leading 15, 15, 12, and 10 EOFs of each respective field. In this diagnostic study, the truncations were chosen through sensitivity analysis and the numerical stability of the LIM, and in the case of SST, the separation of the modes in defining the coupled and internal atmospheric subspaces (see section 4 ). Only $\sim 1 \%$ or less of each field's variance is explained by each additional EOF, indicating that they are not essential to capture the variability of each field. Similar values were also used in Winkler et al. (2001) for heating and extratropical streamfunction. We, however, retained slightly less of the streamfunction variance for numerical stability. We find that non-normal growth is possible in the resulting dynamical operator $\mathbf{L}$, based on the expression $\left\|\mathbf{L}^{\mathrm{T}} \mathbf{L}-\mathbf{L L}^{\mathrm{T}}\right\| /\|\mathbf{L}\|^{2}=0.87$. Note an operator is normal when $\mathbf{L}^{\mathrm{T}} \mathbf{L}=\mathbf{L L}^{\mathrm{T}}$, indicating that its eigenvectors are orthogonal (e.g., Farrell and Ioannou 1996, 1999).

\section{Optimal growth of the PNA pattern}

The optimal initial conditions $\mathbf{p}_{1}$ leading to PNA growth over an interval of $\tau=15$ days, and the corresponding final conditions found by $\mathbf{G}_{15} \mathbf{p}_{1}$, are shown in Fig. 2. Since the optimal patterns and final conditions are based on eigenanalysis, the sign and amplitude of the anomalies are arbitrary but agree between initial and final conditions. As this is a purely diagnostic study, results are scaled by projecting the PNA norm onto the 200-hPa streamfunction component of $\mathbf{x}$ and using the 1.5 standard deviation value of the resulting time series. As there is no "best" way to scale the anomalies, and as the scaling is arbitrary in the first place, we use this scaling for the full and internal space LIM analyses to provide comparable amplitudes of the final $200-\mathrm{hPa}$ streamfunction anomalies. This selected scaling results in amplitudes weaker than that of the PNA pattern composites (Fig. 1), as many of the maps presented herein are only representative of the deterministic component of PNA variability [captured by (2)] and exclude the stochastic [see (1)] contributions to PNA variance. A lag of 15 days is used to allow sufficient time for any influence of tropical heating to impact the full PNA region (e.g., Jin and Hoskins 1995; Matthews et al. 2004; Lin et al. 2009). PNA growth is realized in the streamfunction final condition (Fig. 2a; right panel; see appendix A for discussion on "growth"). In the tropics, any final condition (i.e., Fig. 2b, bottom panel) is not a result of targeted growth toward the final tropical patterns shown (recall the final norm is zeroed in the tropics), but a result of the optimal initial conditions and the interactions between the state vector variables.

The optimal streamfunction anomalies that grow into the PNA pattern after 15 days (Fig. 2a, left panel) show weak amplitude at $850 \mathrm{hPa}$ (gray contours), suggesting that $850-\mathrm{hPa}$ streamfunction does not contribute much to PNA growth. Optimal initial 200-hPa streamfunction conditions (color shading) include positive anomalies over the central Pacific, the northeast Pacific, the central Atlantic, and southeast North America. The evolution of this 15-day optimal is shown in Fig. 3a for days 1, 4, 7, and 10, where the optimal initial condition (Fig. 2a, left panel) is day 0 and the final condition (Fig. 2a, right panel) occurs on day 15 . Figure 3 a shows that the anticyclonic anomaly over the northeast Pacific retrogrades and becomes part of the final PNA pattern, in agreement with the composite evolution of the PNA discussed in other studies (e.g., Franzke et al. 2011; Cash and Lee 2001). Interestingly, this retrogression is consistent with the optimal development of North Pacific blocking (Breeden et al. 2020), suggesting that blocking may play a role in PNA development.

Optimal Q1 initial conditions (Fig. 2b, top panel; color shading) include anomalously suppressed convection across the central Pacific, with the strongest suppression in the region of the South Pacific convergence zone (SPCZ), as well as enhanced convection over the south Indian Ocean and the continental South Atlantic convergence zone (SACZ). Overall, the initial optimal pattern partially resembles both the MJO and ENSO, suggesting that optimal PNA growth from the tropics 
a)
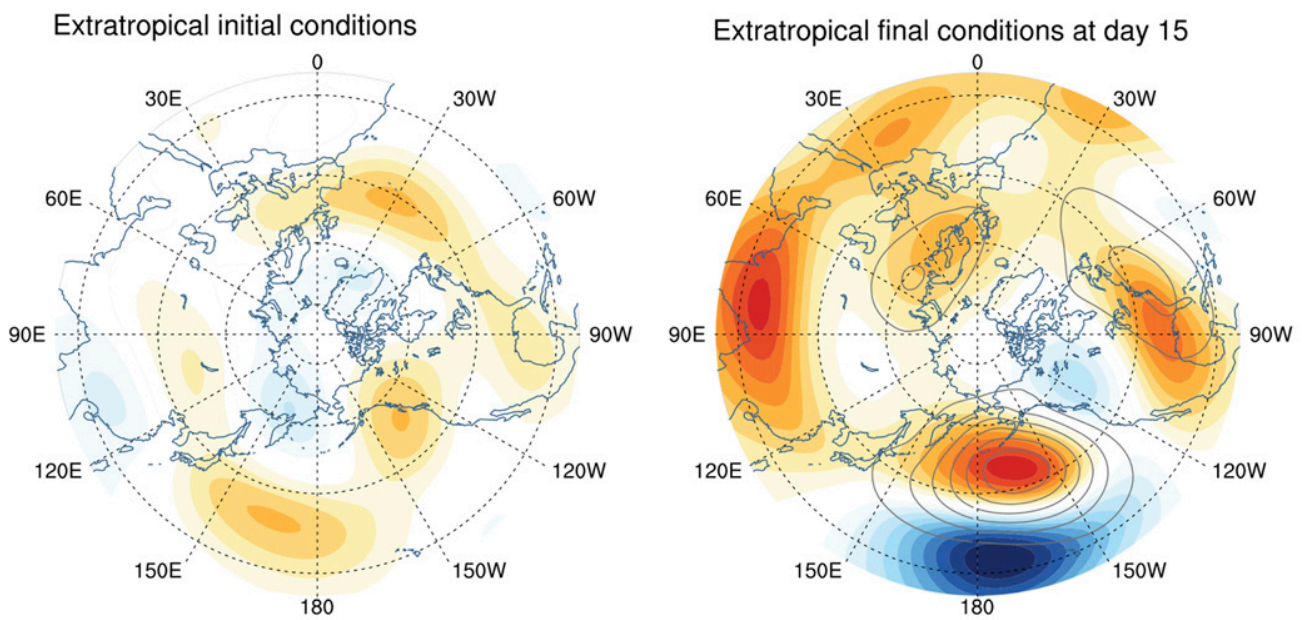

b)
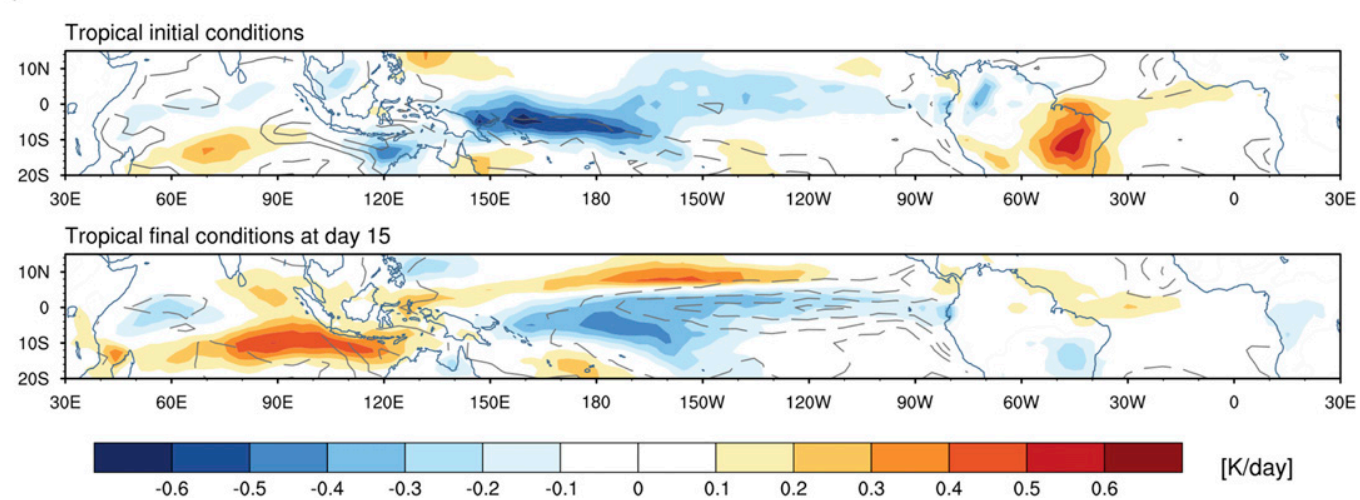

FIG. 2. Optimal initial structure for growth toward a PNA norm and the optimal growth structure into which the initial structure grows into after 15 days for (a) 200-hPa (color shading) and $850-\mathrm{hPa}$ (contours) streamfunction, and (b) vertically integrated Q1 (color shading) and SST (contours). The 850-hPa streamfunction contours are every $1 \times 10^{6} \mathrm{~m}^{2} \mathrm{~s}^{-1}$, and SST contours are every $0.1 \mathrm{~K}$. All negative contours are dashed. Figures are scaled by projecting the PNA norm onto the 200-hPa streamfunction component of $\mathbf{x}$ and using the 1.5 standard deviation value.

may be driven by heating related to both phenomena. This is in part evidenced by the time series of the Q1 optimal (not shown), which peaks during ENSO years and has a correlation of 0.61 with the leading PC of the tropical SST state vector. The time series of the Q1 optimal also has a 0.53 correlation with the third leading $\mathrm{PC}$ of Q1, which has a spectral peak in the intraseasonal time range (see Fig. B2). Employing the RMM indices of Wheeler and Hendon (2004), Q1 composite patterns for each of the eight MJO phases are projected onto the Q1 optimal pattern (not shown). The amplitude of this projection is highest with MJO phases 2 and 6 (recall that the sign of the optimal is arbitrary).

The time-longitude evolution of the $15^{\circ} \mathrm{S}-10^{\circ} \mathrm{N}$ averaged 15-day optimal Q1 (Fig. 3b; color shading) and SST (contours) demonstrates that the anomalous heating over the Indian Ocean propagates eastward within the first 40 days, accompanied by persistent opposite-signed ENSO-like heating and SSTs in the central Pacific. The ENSO pattern is evident in the SST final condition (Fig. 2b, bottom; black contours), which shows negative SST anomalies along the eastern and central equatorial Pacific. Interestingly, the ENSO-like SST anomalies are weak in the initial condition (Fig. 2b) and rapidly amplify; this is due to quickly decaying opposite-signed SST in the internal eigenspace of the full optimal, discussed in section 4. Recall that the scalings of the initial and final patterns are arbitrary though consistent with one another: the growth of SST in Fig. $2 \mathrm{~b}$ is due to the 1.5 sigma scaling used and therefore not typical of SST 
a) 200-hPa streamfunction
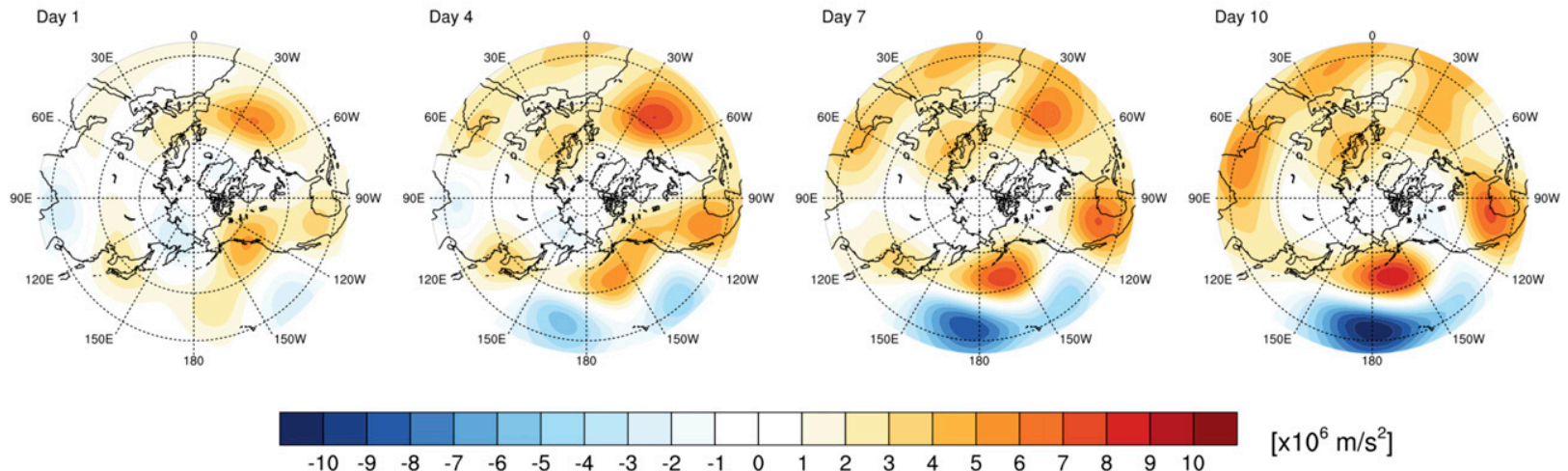

$\left[\times 10^{6} \mathrm{~m} / \mathrm{s}^{2}\right]$

b) Tropical Q1 and SST Hovmöller
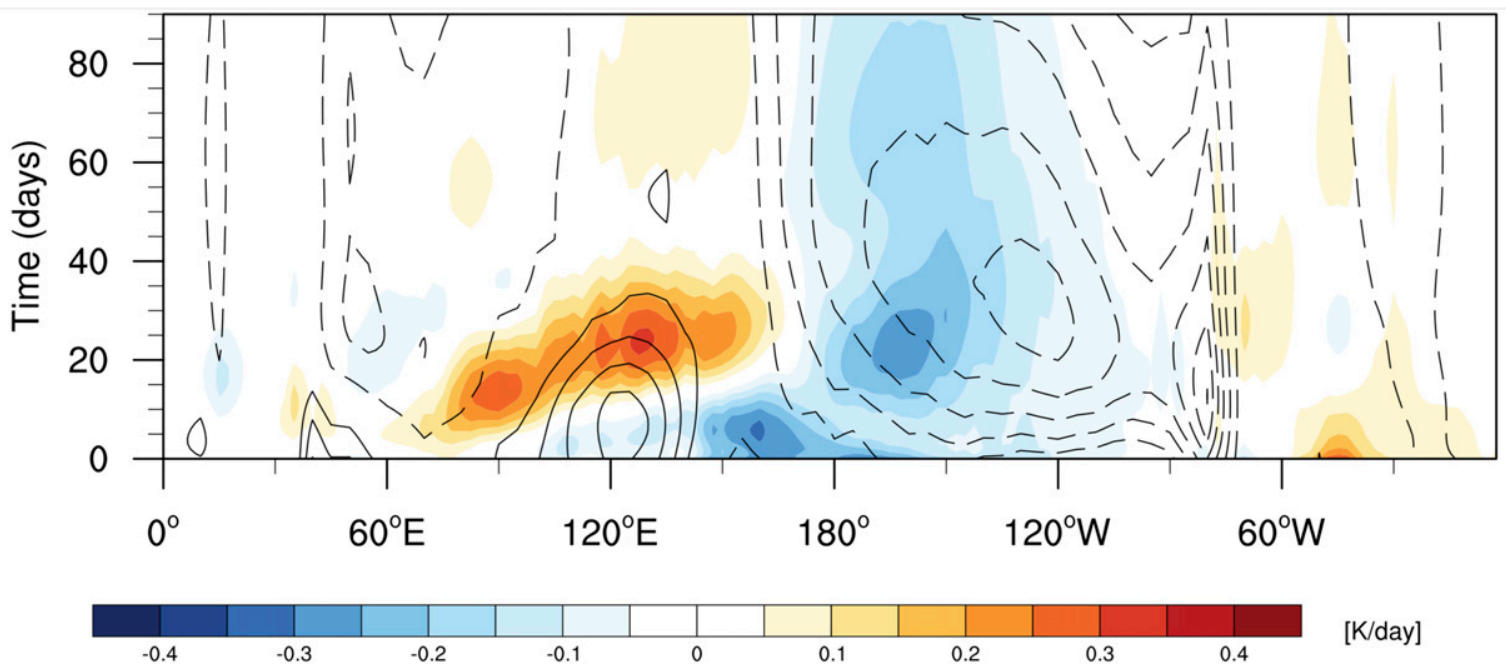

FIG. 3. Evolution of 15-day optimal (a) 200-hPa streamfunction anomalies from (left) day 1 through (right) day 10 (right), and (b) $15^{\circ} \mathrm{S}-10^{\circ} \mathrm{N}$ tropical Q1 (color shading) and SST (contours) from day 0 through day 90 ( $y$ axis). The SST contour interval is $0.05 \mathrm{~K}$.

growth over a 15-day time period. However, we note that rapid large-scale changes in equatorial SST are not unprecedented. For example, during MJO events an average east Pacific SST amplitude change of $\sim 0.4 \mathrm{~K}$ occurs from MJO phase 2 to MJO phase 5 , which is approximately a two-week time period (e.g., Waliser et al. 2009, their Fig. 13). The ENSO signature in the final Q1 pattern is amplified when a longer optimal lag is used, becoming strongest at lags beyond 45 days (i.e., $\mathbf{G}_{45} \mathbf{p}_{1}$; not shown). At such long lags, the initial streamfunction optimal is absent and growth is primarily driven by ENSO, with a final condition resembling the TNH pattern (see also appendix A). This final pattern at longer lags is similar to the final pattern after 14 days following L2 growth in Winkler et al. (2001), which is largely driven by ENSO heating. A key difference, however, is that our inclusion of SST in the state vector lengthens the time scale of development and evolution of ENSO.

It appears that optimal PNA growth is largely dominated by coincident ENSO and MJO variability, implying enhanced PNA predictability during ENSO years. This is suggestive of an interaction between the slowly varying modes that are strongly coupled to SST (e.g., ENSO) and the faster varying modes that are more internal to the atmosphere (e.g., the MJO). We next examine the role of these SST-coupled and internal subspaces in PNA growth to better understand how these subspaces interact and lead to non-normal PNA growth. 


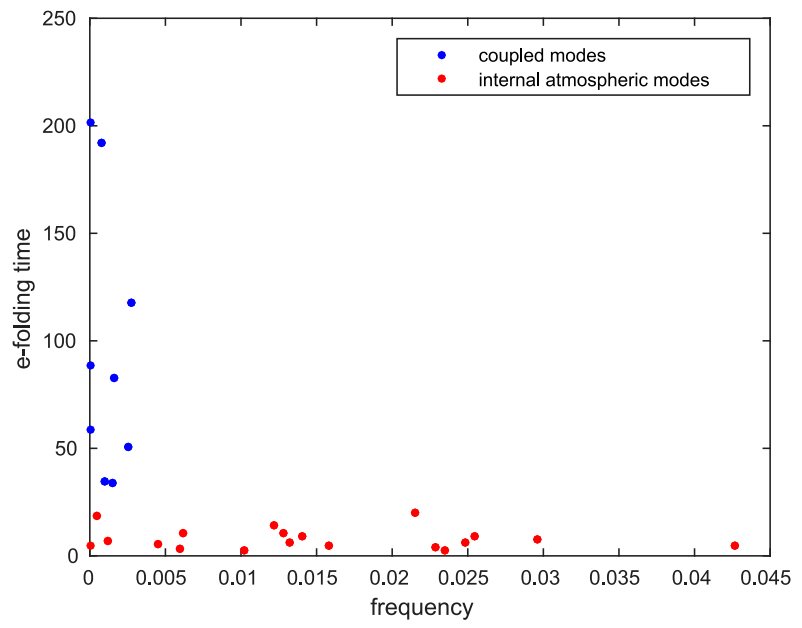

FIG. 4. Frequency $\left(\right.$ day $^{-1}$ ) and $e$-folding time (days) of the eigenvalues of $\mathbf{L}$. Modes strongly coupled to SST are in blue, and internal atmospheric modes are in red.

\section{Internal atmospheric and coupled subspaces of $L$}

\section{Separating the two subspaces}

The eigenvectors of $\mathbf{L}$, sometimes also referred to as principal oscillation patterns (POPs; von Storch et al. 1995), are spatial patterns in $\mathbf{x}$ that occur in complex conjugate pairs with corresponding eigenvalues $\omega$ that indicate the decay and oscillation time scale of the mode. Following Newman et al. (2009), we will demonstrate that the eigenvectors $\mathbf{u}$ of the dynamical operator derived in section 3 using (6) form two separate subspaces consisting of modes either strongly or weakly coupled to SST. The eigenvalues of $\mathbf{L}$ are shown in Fig. 4 as a function of $e$-folding time $\left[y\right.$ axis; $\left.-\operatorname{Re}(\omega)^{-1}\right]$ and frequency $[x$ axis; $\operatorname{Im}(\omega / 2 \pi)]$. Also indicated in the figure are those modes with relatively high SST amplitude versus those modes with relatively little or no SST amplitude. Newman et al. (2009) showed that airsea coupling within the LIM strongly impacted the entire subspace of high SST amplitude eigenmodes (blue circles), which they termed the "coupled" modes. However, the remaining low SST amplitude eigenmodes (red circles) had atmospheric components that were uncoupled or only weakly coupled with SST, so they were called the "internal atmospheric" modes. These modes include the MJO frequency range $\left(\sim 0.01-0.03 \mathrm{day}^{-1}\right)$. Although we have not defined these two subspaces using either frequency or $e$-folding time, Fig. 4 demonstrates that these two subspaces are nevertheless fairly distinct: the coupled modes all have a higher $e$-folding time and have seasonal-to-interannual frequencies, while the internal atmospheric modes have small $e$-folding times and include a wide range of frequencies. This overall picture is similar to Fig. 9 of Newman et al. (2009).
Together, the coupled and internal atmospheric subspaces must (by construction) entirely span the full state space of $\mathbf{x}$. Therefore, the optimal initial conditions leading to PNA growth in the full LIM ( $\mathbf{p}_{1} ;$ Fig. 2) can be decomposed into its coupled and internal atmospheric components. The full optimal initial condition can be written as a summation across all $j$ eigenvectors of $\mathbf{L}$ (e.g., Penland and Matrosova 2006; Newman et al. 2009):

$$
\mathbf{p}_{1}=\sum_{j} \mathbf{u}_{j} \alpha_{j}^{p}
$$

where $\mathbf{u}_{j}$ contains the eigenvectors of $\mathbf{L}$, and $\alpha_{j}^{p}$ is the inner product of $\mathbf{p}_{1}$ with the corresponding adjoint vectors of $\mathbf{u}_{j}$. The projection onto the adjoint vectors is necessary since the $\mathbf{u}_{j}$ vectors are not orthogonal. The internal atmospheric component of the full optimal initial condition $\mathbf{p}_{1}^{\text {int }}$ is obtained by summing across only the internal atmospheric modes (Fig. 4, red circles); similarly, the coupled component $\mathbf{p}_{1}^{\text {coup }}$ is the sum of only the coupled modes. The final conditions associated with each of the internal atmospheric and coupled components are found by evolving them separately using the full $\mathbf{G}$ from (4).

The internal atmospheric and coupled components of the full LIM optimal initial conditions and the associated final structures are shown in Figs. 5 and 6 for the tropical fields, and 200-hPa streamfunction, respectively. As in previous figures, all amplitudes are scaled using the 1.5 standard deviation value of the projection of the PNA norm on the $200-\mathrm{hPa}$ streamfunction component of $\mathbf{x}$. Recall this scaling is arbitrarily chosen for this diagnostic study and may not indicative of, for example, real amplitudes associated with ENSO anomalies, which would be specific to the coupled space. SST initial conditions in the two subspaces (Fig. 5a; black contours) include opposite-sign SSTs in the equatorial Pacific that largely cancel, leading to the weak SST initial conditions shown in Fig. 2b (top panel). The positive SST anomalies in the internal atmospheric space quickly decay, whereas the negative SST anomalies in the coupled space persist and are apparent in the coupled space 15 days later (Fig. 5b, bottom). Recall the internal atmospheric space includes some eigenmodes that are weakly coupled to SST. These internal modes will have some SST response, although in many cases this response has little impact on the evolution of the atmospheric component of the mode (Newman et al. 2009). For example, the internal atmospheric space includes an SST response to the MJO (Fig. 8). However, the separation of the two subspaces based on SST amplitude indicates that the coupling to SST does not greatly impact the internal atmospheric modes. We also note that while the scaling was arbitrarily chosen, the internal 
a) Initial conditions
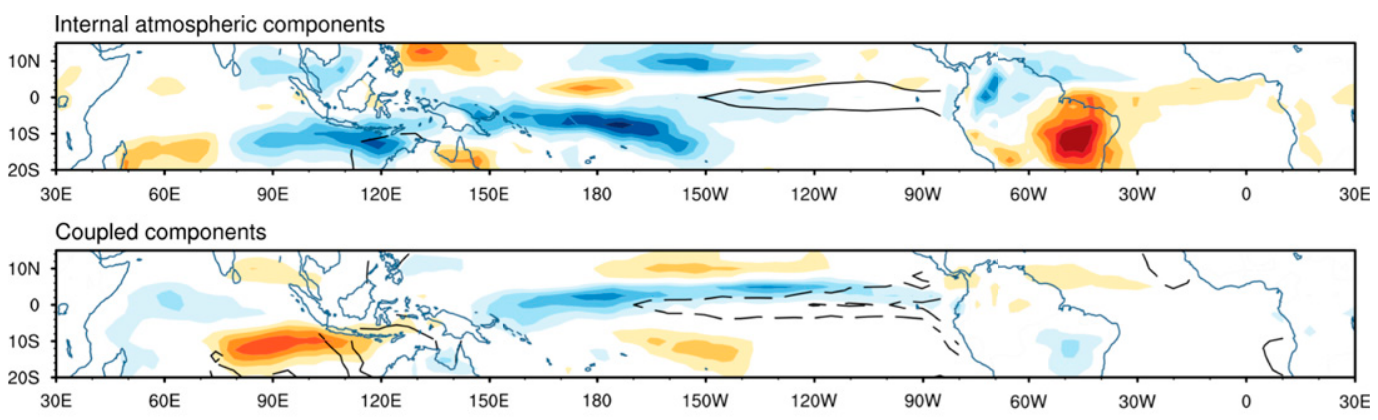

b) Final conditions

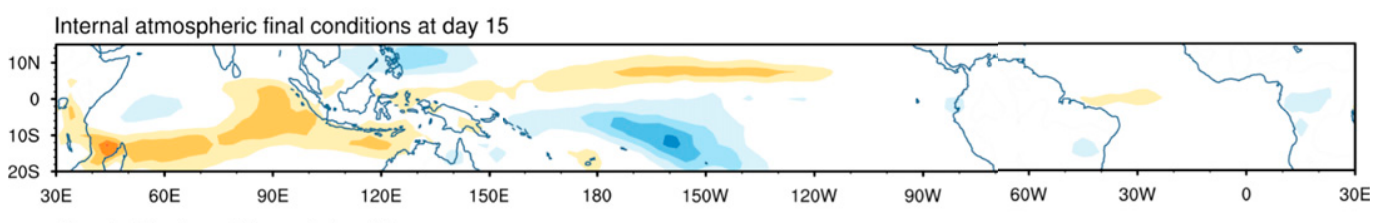

Coupled final conditions at day 15

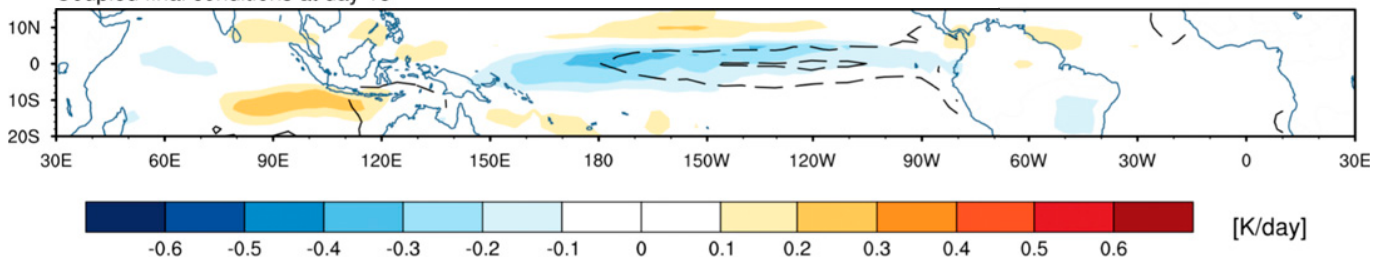

FIG. 5. Internal atmospheric and coupled components of (a) the full SST (black contours) and Q1 (color shading) initial conditions and (b) the final structure 15 days later, found by evolving each component separately using the full G. SST contours are every $0.2 \mathrm{~K}$ and negative values are dashed.

atmospheric SST anomaly amplitude in the east Pacific (Fig. 5a, top) is realistic for an MJO event (e.g., Waliser et al. 2009, their Fig. 14).

Tropical Q1 (Fig. 5a; color shading) and 200-hPa streamfunction (Fig. 6a) also demonstrate oppositesigned anomalies in the optimal initial internal atmospheric components relative to the coupled components; similar opposite-signed behavior is seen for $850-\mathrm{hPa}$ streamfunction (not shown). This counterintuitive result indicates that the greatest PNA growth occurs when the internal atmospheric and coupled components of the initial conditions interfere such that they largely cancel out, minimizing the amplitude of the optimal initial conditions shown in Fig. 2. There are some key differences, however. Final conditions show that Indian Ocean heating primarily evolves in the internal atmospheric space (Fig. 5b, top), whereas equatorial Pacific heating and SSTs persist only in the coupled space (Fig. 5b, bottom). PNA growth occurs in both the internal atmospheric and coupled spaces (Fig. 6b), although the coupled PNA growth (Fig. 6b, right panel) evolves from a similar PNA-like pattern of slightly higher amplitude (Fig. 6a, right panel), indicative of the slower low-frequency variability of the coupled subspace (e.g., Fig. 4). The anomalies in the coupled space are also overall weaker relative to the internal atmospheric space, suggesting that PNA growth primarily occurs in the internal atmospheric space (this is in agreement with Fig. 7 and Fig. A1, right panel). The coupled space primarily evolves at longer time scales. When a 45-day lag is used instead of a 15-day lag (not shown), growth occurs only in the coupled space for all fields, with a strong ENSO signature in the final SSTs and Q1, and a 200-hPa streamfunction final pattern that more closely resembles the TNH pattern over the Pacific Ocean (see also appendix A).

The large cancellation between contributions of the coupled and internal atmospheric subspaces of $\mathbf{L}$ in the optimal initial conditions (Figs. 5 and 6) is an important result that warrants further discussion. As growth is defined relative to the amplitude of the initial state, it appears that the internal atmospheric space is largely negating initial anomalies in the more persistent coupled space. Growth, then, occurs as the fast-varying structures in the internal atmospheric space decay, leaving the slowly decaying structures in the coupled space, in 
a) Initial conditions

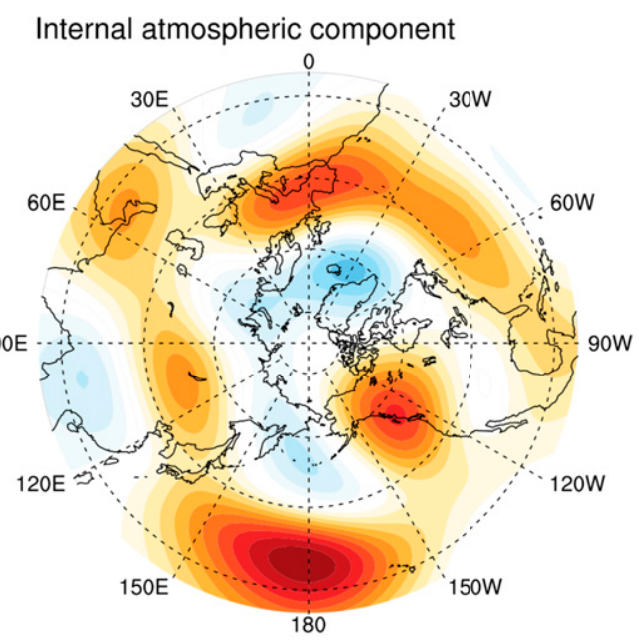

b) Final conditions

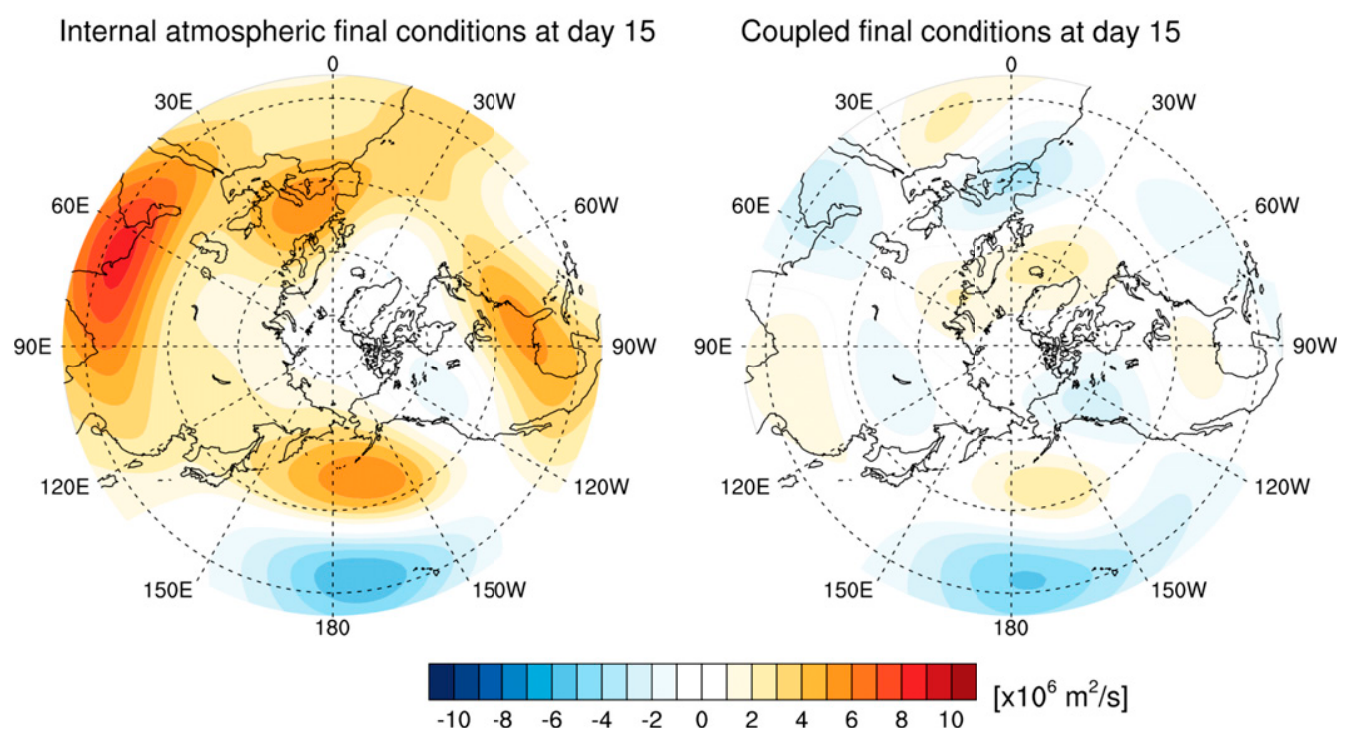

FIG. 6. Internal atmospheric and coupled components of (a) the full PSI 200 initial conditions and (b) the final

structure 15 days later, found by evolving each component separately using the full $\mathbf{G}$.

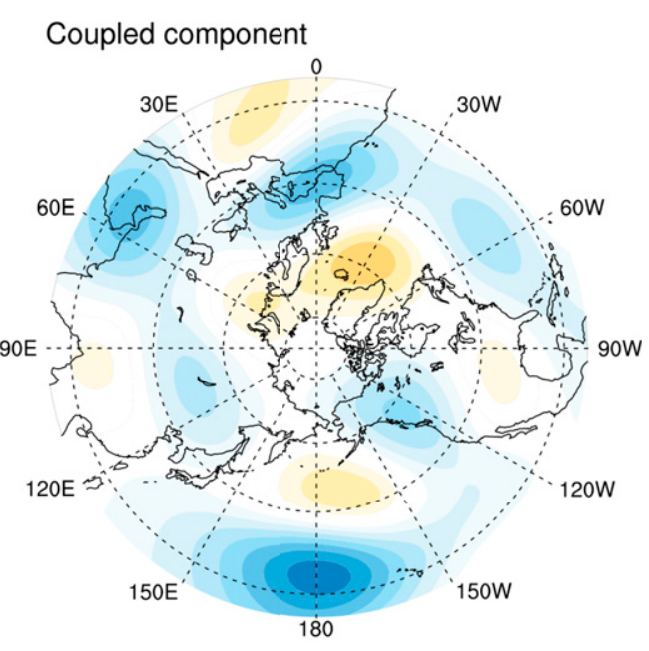

part evidenced by the changes in amplitude of the initial and final streamfunction patterns in the PNA region (Fig. 6). This means that diagnosing the internal atmospheric contribution to the full optimal may not yield physically meaningful information about how PNA development optimally occurs in the absence of oceanatmosphere coupling. This is a consequence of the nonnormality of the eigenmodes, the choice of norm used to define growth, and the calculation of the optimal under the full (coupled plus internal atmospheric) initial space. The internal atmospheric subspace contribution to PNA growth may be better assessed with the development of an independent internal atmospheric LIM that excludes the coupled space.

\section{The internal atmospheric LIM}

Development of a LIM that excludes strongly SSTcoupled modes, referred to here as the "internal atmospheric" LIM, consists of two main stages. First, we filter $\mathbf{x}$ by removing its projection on all of the coupled modes determined from the full dynamical operator $\mathbf{L}$, which include the modes representing ENSO variability. This results in a new internal atmospheric state vector that 


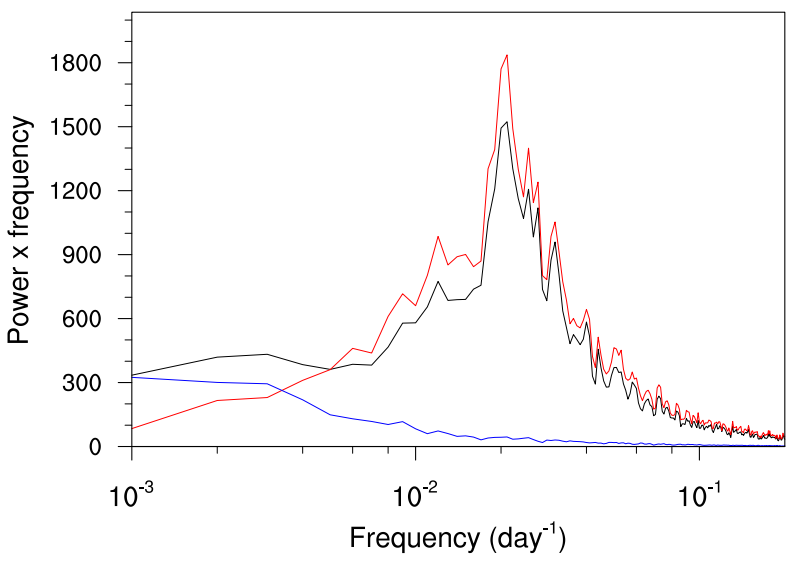

FIG. 7. Power spectra of the PNA index calculated using the full PCs are shown in black. Also shown are the spectra of the PCs filtered by summing over the internal atmospheric modes of $\mathbf{L}$ (red curves) and by summing over the coupled modes of $\mathbf{L}$ (blue curves). See text for details.

contains variability generated only by dynamics internal to the atmosphere, $\mathbf{x}^{\text {int }}$, from which we then develop an independent LIM, repeating (1)-(5). The development of such an internal atmospheric LIM has not been previously done to the best of the authors' knowledge. The internal atmospheric LIM will allow us to examine the optimal conditions that lead to PNA growth outside of the influence of ENSO.

Decomposing the state vector is done by defining $\mathbf{x}$ in terms of the eigenvectors of $\mathbf{L}$ in the same manner as previously discussed for the optimal full initial conditions (see section 4). Following the methodology of Penland and Matrosova (2006) and Newman et al. (2009), we define

$$
\mathbf{x}(t)=\sum_{j} \mathbf{u}_{j} \alpha(t),
$$

where $\mathbf{u}_{j}$ are the eigenvectors of $\mathbf{L}$, and the corresponding adjoints $\mathbf{v}_{j}$ are used to calculate $\alpha(t)=\mathbf{v}_{j}^{\mathrm{T}} \mathbf{x}(t)$. The subscript $j$ indicates that the sum in (8) is across all 52 modes of $\mathbf{L}$. The state vector is divided into coupled $\mathbf{x}^{\text {coup }}$ and internal atmospheric components $\mathbf{x}^{\text {int }}$, where $\mathbf{x}^{\text {coup }}$ is calculated using (8) but summing only over the coupled modes. The PCs containing the internal atmospheric (i.e., uncoupled or weakly SST-coupled) variability $\mathbf{x}^{\text {int }}$ are then the residual $\mathbf{x}^{\text {int }}=\mathbf{x}-\mathbf{x}^{\text {coup }}$. This separation acts as a filter based on the dynamics of the system, and cleanly separates strong SST-coupled variability such as ENSO (including diverse "types" of ENSO events; Capotondi et al. 2015) from internal atmospheric variability like the $\mathrm{MJO}$ without requiring frequency cutoffs or edge effects (e.g., Newman et al. 2009; also, see appendix B herein for testing of the filter).
Last, we revert $\mathbf{x}^{\text {int }}$ from PC space to physical space to obtain an "internal atmospheric" dataset for each field. These internal atmospheric datasets are then used in section $5 b$ to develop the internal atmospheric LIM.

\section{a. Non-normality of the internal atmospheric and coupled spaces}

An important aspect of the coupled and internal atmospheric spaces is the non-normality between these two spaces. This is examined for the PNA index here, and is further explored in appendix B. Power spectra of the PNA index for the full, internal atmospheric, and coupled state vector components are shown in Fig. 7 in black, red, and blue, respectively. The power spectra are calculated using a 20000-day forward integration of (1) and summing over all 52 modes, the internal atmospheric modes, or the coupled modes of the full $\mathbf{L}$ (i.e., Fig. 4) to obtain a long run of $\mathbf{x}, \mathbf{x}^{\text {int }}$, and $\mathbf{x}^{\text {coup }}$, respectively. A long-run PNA index is then computed by projecting the PNA norm, $\mathbf{r}_{\mathrm{PNA}}$ from (5), onto these 20000 -day time series. A description of the forward integration method is provided in appendix B and follows the methodology of Penland and Matrosova (1994). As in Fig. B2, the time series are subdivided into overlapping segments and a Hann window is applied (see appendix B for details). Two things are evident from Fig. 7. One is that the majority of the PNA variance is in the intraseasonal time range, which is captured primarily by the internal atmospheric space. The other is that when summing the internal atmospheric (red) and coupled (blue) spectra, the power exceeds that of the full state vector (black), with the internal atmospheric power spectra often exceeding that of the full. This is a result of the non-normality between the coupled and internal atmospheric spaces. In fact, this is clear when examining the internal atmospheric and coupled spaces beyond the PNA index, as evidenced and discussed in appendix B. This non-normality means that diagnosing the two subspaces would be difficult without a LIM-for example, a high-pass temporal filter would still leave a coupled space component-making LIM the ideal tool to examine PNA development in the internal atmospheric space independent from strong SST-coupled variability like ENSO.

\section{b. Internal atmospheric optimal growth}

A 26-component internal atmospheric state vector is generated using an upper bound of $88 \%$ variance retained for all fields, comprising the leading 11, 8, and 7 EOFs of the internal atmospheric tropical Q1, extratropical PSI ${ }_{200}$, and extratropical PSI $_{850}$ datasets and explaining approximately $88 \%, 84 \%$, and $87 \%$ of the variance, respectively. A higher percentage of the variance is explained by a fewer number of EOFs relative to (6) due to the filter. The 


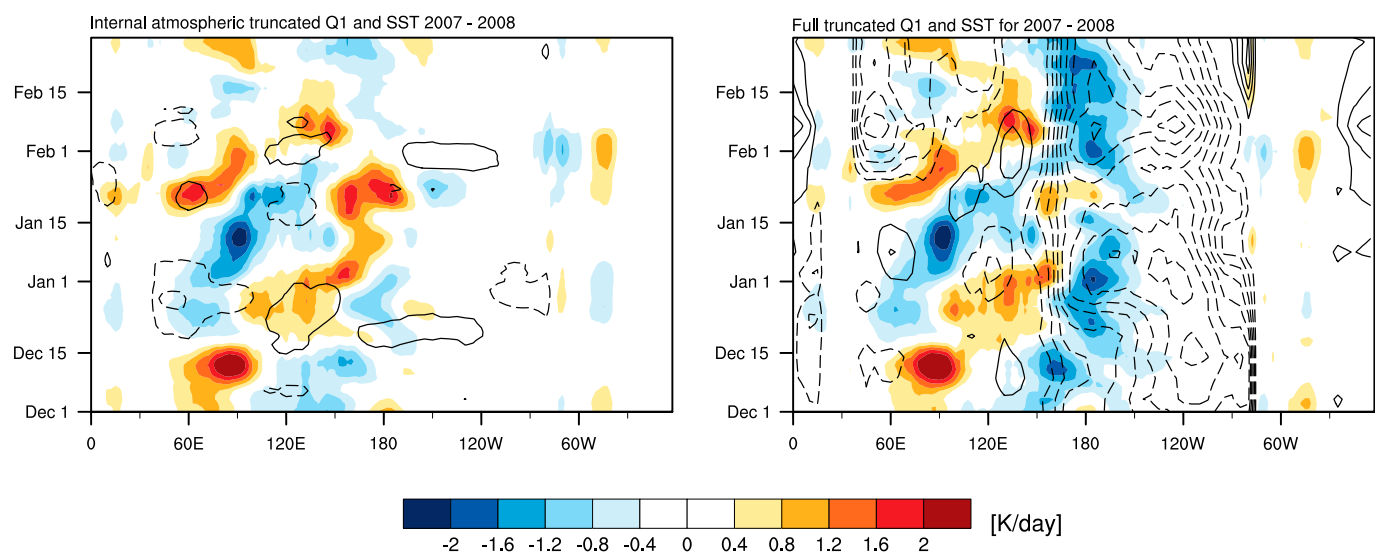

FIG. 8. Hovmöller diagrams of $10^{\circ} \mathrm{S}-5^{\circ} \mathrm{N}$ averaged EOF-truncated SST (black contours) and EOF-truncated Q1 (color shading) for the 2007/08 DJF season using the (left) internal atmospheric and (right) full state vector data. SST contours are every $0.2 \mathrm{~K}$ and negative values are dashed.

variance retained for each dataset is determined to be the maximum threshold yielding a numerically stable LIM. The internal atmospheric LIM is derived in the same manner as the full LIM previously discussed [i.e., Eqs. (1)(5)], except that the 26-component internal atmospheric state vector is used instead of the full 52-component state vector in (6). Unlike in the full LIM, SST is excluded from the internal atmospheric LIM state vector. The resulting internal atmospheric dynamical operator is non-normal $\left(\left\|\mathbf{L}^{\mathrm{T}} \mathbf{L}-\mathbf{L L}^{\mathrm{T}}\right\| /\|\mathbf{L}\|^{2}=0.72\right)$, indicating that non-normal growth is possible within the internal space alone.

To demonstrate the effectiveness of this filter in removing strong SST-coupled variability, Fig. 8 shows the (EOF-truncated) Q1 (color shading) and SST (black contours; $87 \%$ variance retained) in the internal atmospheric space (left panel) and in the full space (right panel) for the 2007-08 DJF season, which was characterized by a La Niña event and an active MJO. The SST Hovmöller diagrams demonstrate that the negative SST anomalies associated with La Niña (right panel) are filtered out in the internal atmospheric SST (left panel), as are the heating anomalies associated with these La Niña SST anomalies. This is most evident toward the end of the DJF season when the ENSO heating anomalies are amplified (right panel; e.g., Henderson et al. 2018, see their Fig. 13). Recall that the internal atmospheric space is not fully uncoupled from SST. While the ENSO anomalies are removed in the internal atmospheric Q1 and SST, the eastward propagating heating and SST anomalies associated with the MJO remain, in agreement with the analysis presented in appendix B. Dole et al. (2018) obtained similar results applying this approach on four post-1982 El Niño events.

The optimal initial conditions in the internal atmospheric LIM and the corresponding final conditions after
15 days are shown in Fig. 9 for the extratropical fields and tropical Q1. As in the full LIM, results are scaled by projecting the PNA norm onto the $200-\mathrm{hPa}$ streamfunction component of the full state vector $\mathbf{x}$ and using the 1.5 standard deviation value of the resulting time series. This results in comparable amplitudes to the full LIM. The optimal initial 200-hPa streamfunction conditions (Fig. 9a, left) over the Pacific Ocean are very similar to those using the full LIM (Fig. 2a, left), and by 15 days (Fig. 9a, right) have similarly evolved with a retraction of the optimal initial northeast Pacific anomaly leading to the final PNA pattern (Fig. 10a; cf. Fig. 3a). This suggests that the evolution of the optimal extratropical anomalies in the full LIM is primarily associated with dynamics internal to the atmosphere and not dynamics strongly coupled to SST, such as ENSO. Although atmospheric blocking is not examined here, it is notable that the optimal initial conditions (Fig. 9a, left) and the evolution of the extratropical streamfunction are again similar to optimal North Pacific blocking development (Breeden et al. 2020).

In the tropics, enhanced heating over the Indian Ocean and suppressed heating over the Maritime Continent and SPCZ lead to optimal PNA growth after 15 days (Fig. 9b, top). By day 15 both features have shifted eastward (Fig. 9b, bottom), indicating that the initial Q1 optimal is likely associated with the MJO. This is further evidenced by the time-longitude evolution of Q1 (Fig. 10b), which shows anomalous heating in the east Indian Ocean propagating eastward with time. This is similar to the full LIM Q1 evolution (Fig. 3b) absent the anomalous ENSO heating in the central Pacific. These results agree with those of Mori and Watanabe (2008), who examined the link between the MJO and the PNA pattern. Through examination of the Rossby wave source, their study 
a)
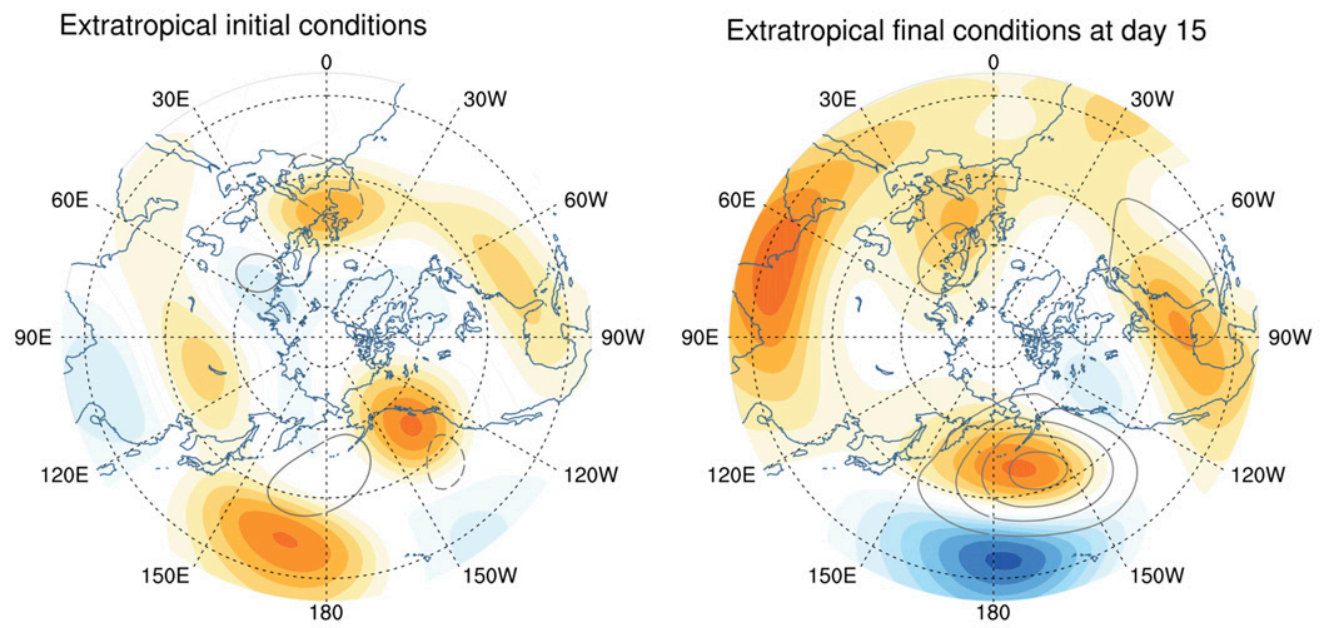

b)
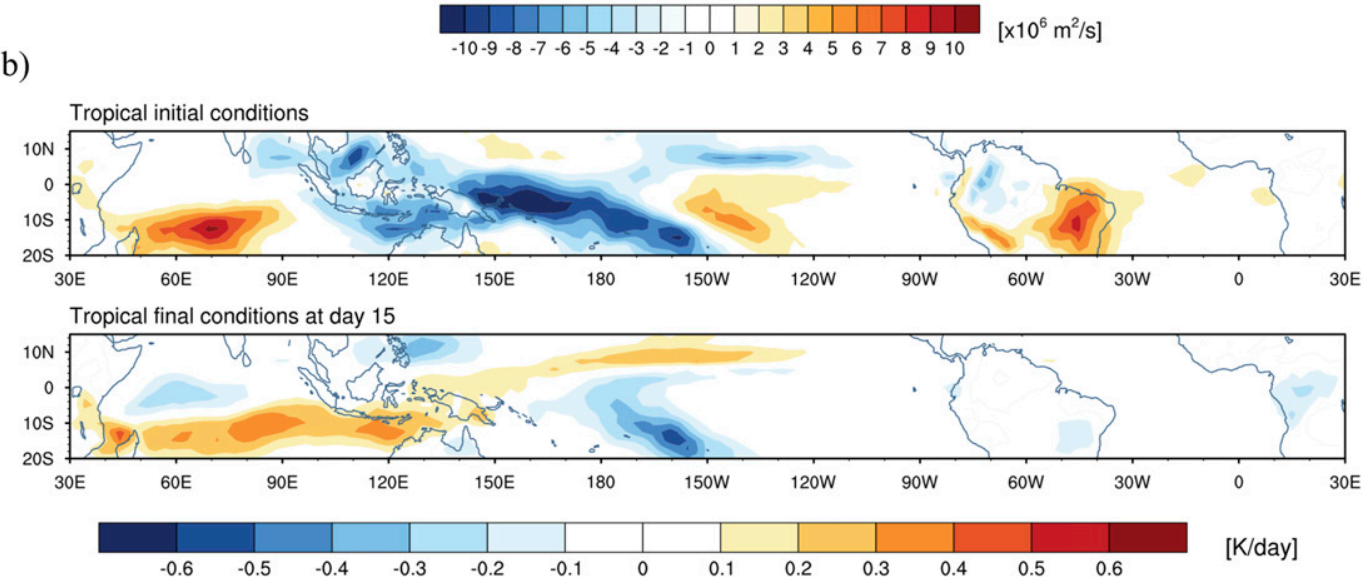

FIG. 9. As in Fig. 2, but using the internal atmospheric LIM. The 850-hPa streamfunction contours are every $1 \times$ $10^{6} \mathrm{~m}^{2} \mathrm{~s}^{-1}$. SST is excluded in the internal atmospheric LIM. As in the full LIM, internal atmospheric LIM figures are scaled by projecting the PNA norm onto the $200-\mathrm{hPa}$ streamfunction component of the full state vector $\mathbf{x}$ and using the 1.5 standard deviation value of the resulting time series.

suggested that divergence associated with MJO heating triggers a PNA pattern through the excitation of a Rossby wave train initialized over the northern Bay of Bengal. Furthermore, Henderson et al. (2016) found that a negative PNA pattern preceding European blocking events was preceded by strong MJO heating over the Indian Ocean (see their Fig. 9). This link is strengthened by our results, suggesting that MJO Indian Ocean heating optimally leads to PNA growth.

LIM analysis shows only what could optimally grow the PNA pattern in a linear sense. It does not mean that optimal growth actually occurs or that the observed dynamics behave in a linear manner. These are tested for the full (Fig. 11, left panel) and internal atmospheric (right panel) LIMs using scatterplots of the projection of the full and internal atmospheric state vector onto each corresponding optimal, $\mathbf{p}_{1}$ ( $x$ axis), versus the projection of each state vector 15 days later onto the PNA norm, $\mathbf{r}_{\text {PNA }}(y$ axis). Both scatterplots have a positive slope and demonstrate a linear relationship, with a fractional variance $r^{2}$ of 0.35 for the full LIM and 0.17 for the internal atmospheric LIM based on a linear regression. Figure 11 indicates that optimal PNA growth does actually occur. Overall, optimal PNA growth is better represented by the full LIM than the internal atmospheric LIM, indicating better PNA pattern predictability during ENSO years. There is scatter, which is expected given that the noise in (1) will play a role in the development of the PNA pattern. For the full LIM, a similar $r^{2}$ is obtained when the optimal initial SST is not used in the projection and only Q1 and streamfunction are considered. However, the $r^{2}$ decreases when only the 
a) $200-\mathrm{hPa}$ streamfunction
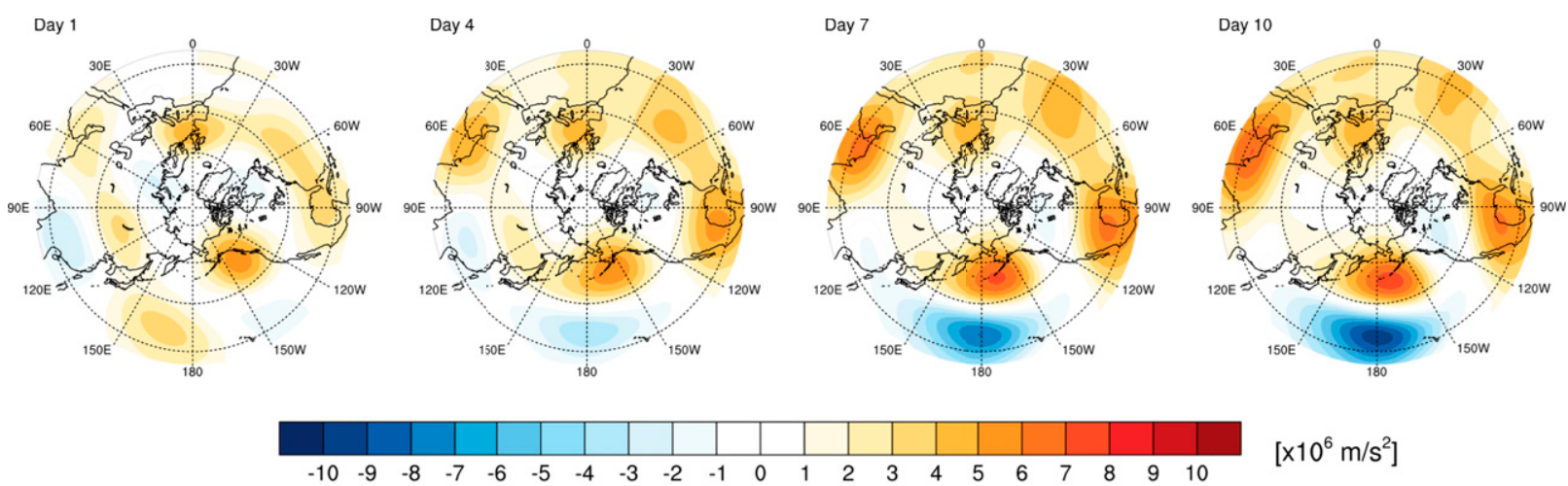

b) Tropical Q1 Hovmöller
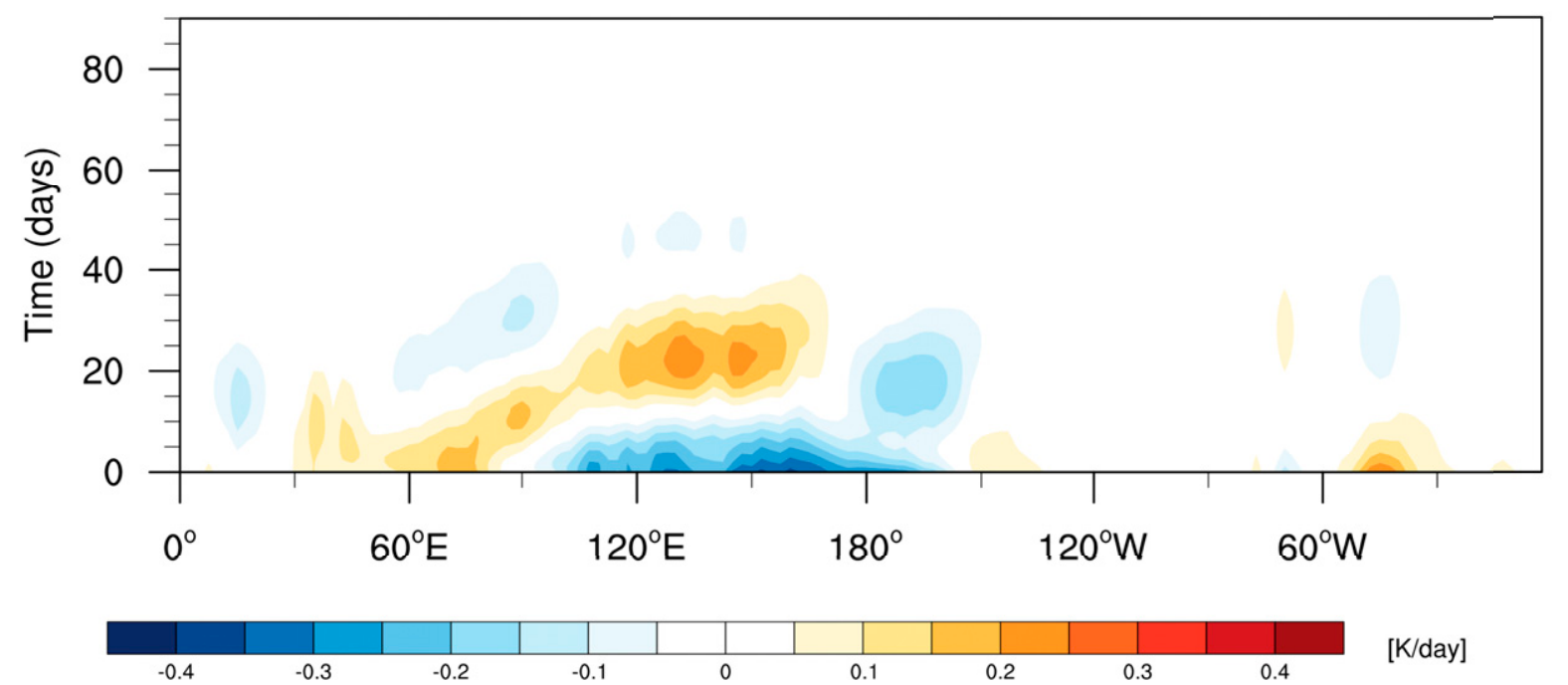

FIG. 10. As in Fig. 3, but for the internal atmospheric LIM. SST is excluded in the internal atmospheric LIM.

Q1 optimal is used $\left(r^{2}=0.23\right)$ or only the extratropical streamfunction optimal initial conditions are used $\left(r^{2}=\right.$ 0.18). Similarly, $r^{2}$ decreases in the internal atmospheric LIM when only the Q1 optimal is considered $\left(r^{2}=0.09\right)$ or only the extratropical streamfunction optimal conditions are considered $\left(r^{2}=0.1\right)$. This suggests improved PNA pattern predictability when both tropical heating and the extratropical circulation are considered, in agreement with appendix A.

\section{Tropical and extratropical contributions to PNA growth}

The LIM analysis thus far has explored the relative importance of SST-coupled versus internal atmospheric dynamics in driving PNA growth, given optimal initial conditions defined throughout the tropics and extratropics. In this section, we further diagnose optimal
PNA growth by quantifying the relative importance of the tropical versus extratropical portions of these initial anomalies themselves.

The tropical contribution to optimal PNA growth is found by zeroing out the extratropical circulation contribution to the optimal initial conditions $\mathbf{p}_{1}$ in (4) and propagating the modified $\mathbf{p}_{1}^{\prime}$ forward via $\mathbf{G}_{\tau} \mathbf{p}_{1}^{\prime}$, with the calculation carried out separately for the full and internal atmospheric LIMs. We refer to this modification of the optimal initial conditions as NIE (for "no initial extratropics"). Likewise, the extratropical contribution is found by instead zeroing out the tropical contribution to $\mathbf{p}_{1}$, referenced here as NIT ("no initial tropics"). Note that this technique removes the contribution of extratropical or tropical variability from the initial condition only. Interactions between the extratropics and tropics still occur through the off-diagonal elements of the dynamical operator $\mathbf{L}$. For example, even with no initial 
a) Full $\operatorname{LIM}\left(r^{2}=0.35, \lambda_{1}=1.46\right)$

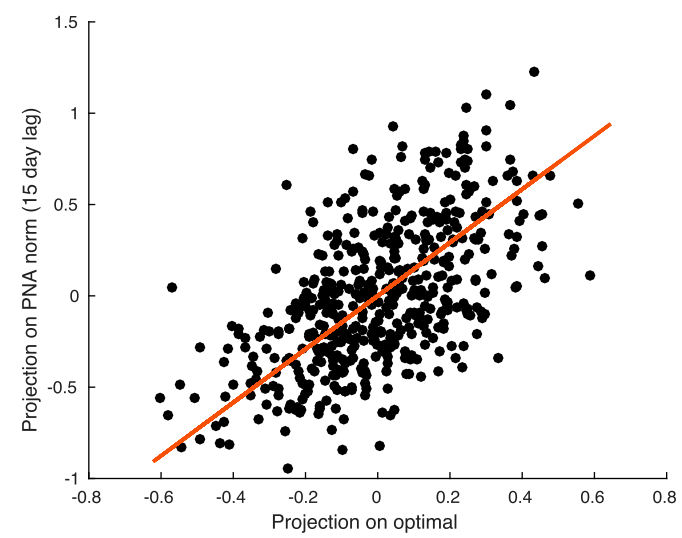

b) Internal atmospheric $\operatorname{LIM}\left(r^{2}=0.17, \lambda_{1}=0.93\right)$

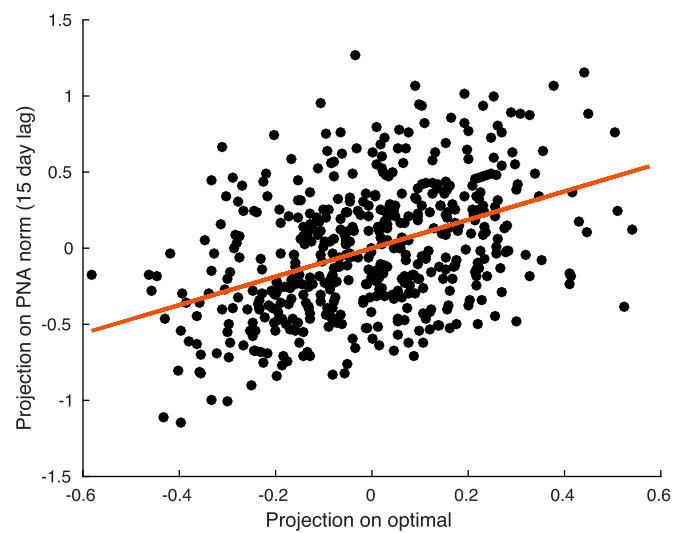

FIG. 11. Scatterplot of the projection of (a) the full LIM and (b) the internal atmospheric LIM state vector onto the optimal ( $x$ axis) vs the projection of each corresponding state vector onto the PNA norm 15 days later. For clarity, only every 5 days are shown. The leading eigenvalue $\lambda_{1}$ corresponding to $\mathbf{p}_{1}$ is represented by the slope of the red line. The corresponding fractional variance $r^{2}$ and $\lambda_{1}$ are shown above each panel.

tropical conditions, the extratropical initial condition can lead to tropical heating anomalies, which can, in turn, influence PNA growth. This relationship will be addressed later. Growth curves for these modified initial conditions are provided in appendix A.

Final patterns after 15 days using the NIE and NIT modified optimal initial conditions are shown in Fig. 12 for the full LIM. When the initial extratropical conditions are removed (NIE), the final PNA pattern at day 15 (Fig. 12a, left) is weakened relative to that shown in Fig. 2a (right). Greater weakening of the $200-\mathrm{hPa}$ streamfunction final condition is observed for NIT (Fig. 12a, right), suggesting that the lack of ENSO and strong MJO heating leads to a much weaker PNA pattern. In addition to the differences in the extratropical final patterns, there are interesting differences in the final Q1 conditions in both the NIE and NIT cases (Fig. 12b) relative to the unmodified full LIM (Fig. 2b, bottom). The NIE final Q1 pattern suggests that removing the extratropical initial conditions may be weakening the final tropical heating (Fig. 12b; top), and the NIT final Q1 pattern indicates that the initial extratropical circulation may be partially enhancing the Indian Ocean heating and suppressing heating over the west Pacific Ocean (Fig. 12b; bottom). The impact of the extratropical circulation on tropical heating is beyond the scope of this study and is a subject of ongoing work. However, for NIT, it is possible that the weak tropical heating that still develops (Fig. 12b, bottom panel) is forcing some of the final PNA pattern shown. We test this by setting to zero the off-diagonal elements of $\mathbf{L}$ that characterize the impact of the tropics onto the extratropics (e.g., Newman and Sardeshmukh 2008). The final PNA pattern from this additional modification (not shown) has similar amplitude as that shown in Fig. 12a (right) with a slightly weaker Pacific cyclonic anomaly, demonstrating that the optimal initial extratropical circulation (i.e., Fig. 2a, left) is primarily responsible for the PNA growth shown in Fig. 12. It is also worth noting that the ENSO pattern in the final condition is absent in Fig. 12b (bottom panel), suggesting that the tropical initial conditions are necessary to force to the ENSO anomalies.

We repeat the process above to examine the relative roles of the optimal extratropical and tropical initial conditions in producing 15-day PNA growth in the internal atmospheric LIM (Fig. 13). For NIE, the final 200-hPa streamfunction (Fig. 13a, left) is much weaker relative to the unmodified results (Fig. 9a, right), meaning that the preceding extratropical circulation is important for PNA growth. The tropical Indo-Pacific heating is also important for optimal PNA growth, as shown by a weakened PNA pattern when instead the tropical initial conditions are removed (NIT; Fig. 13a, right). Although the amplitudes are fairly similar, growth in the PNA region is slightly greater for NIT, suggesting a slightly higher contribution from the extratropical initial conditions.

As observed in the full LIM, the final Q1 conditions in the internal atmospheric LIM are also impacted when modifying the optimal initial conditions (Fig. 13b) and are a subject of ongoing work. For NIT, we again test if the final PNA pattern may be in part due to the tropical heating anomalies that develop from the extratropical initial conditions. As done for the full LIM, this is examined by zeroing the off-diagonal elements of $\mathbf{L}$ that characterize the impact of the tropics onto the extratropics. Again, the final PNA pattern (not shown) has similar amplitude as Fig. 13a (right) but with a weaker cyclonic anomaly in the subtropical Pacific. This indicates 
a)
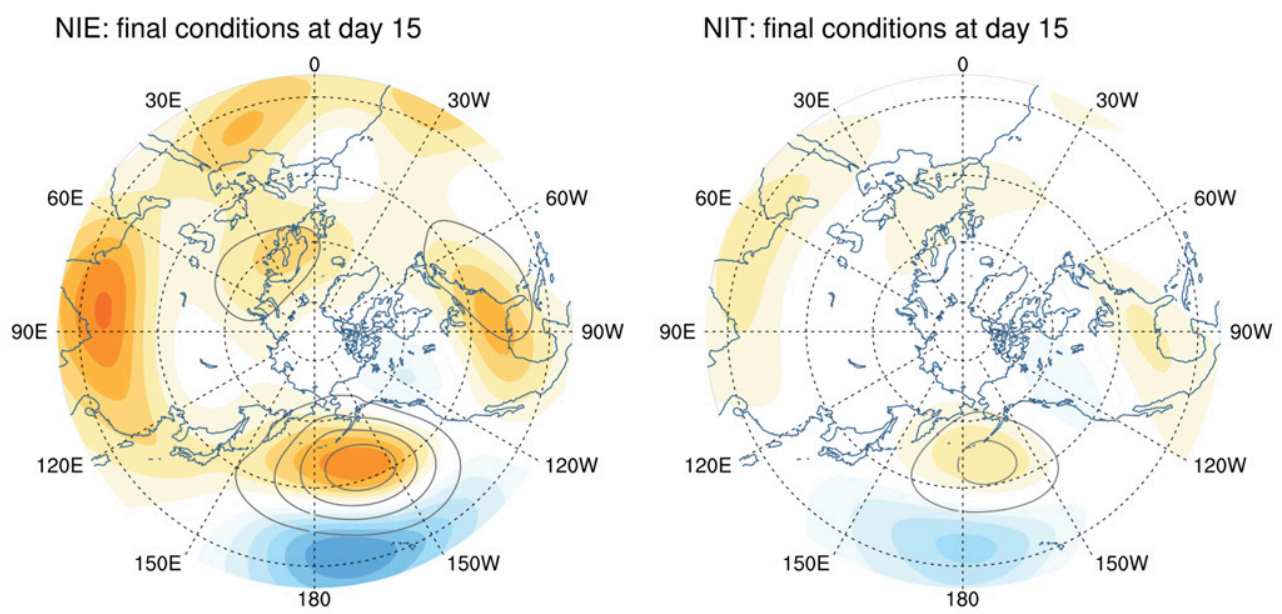

b)

$\left[\times 10^{6} \mathrm{~m}^{2} / \mathrm{s}\right]$

NIE: final conditions at day 15

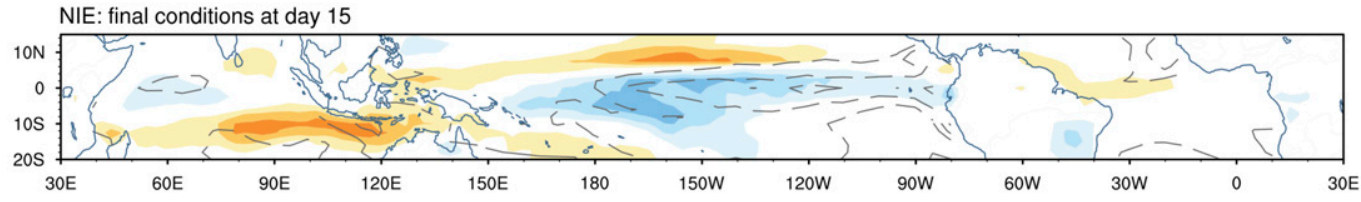

NIT: final conditions at day 15

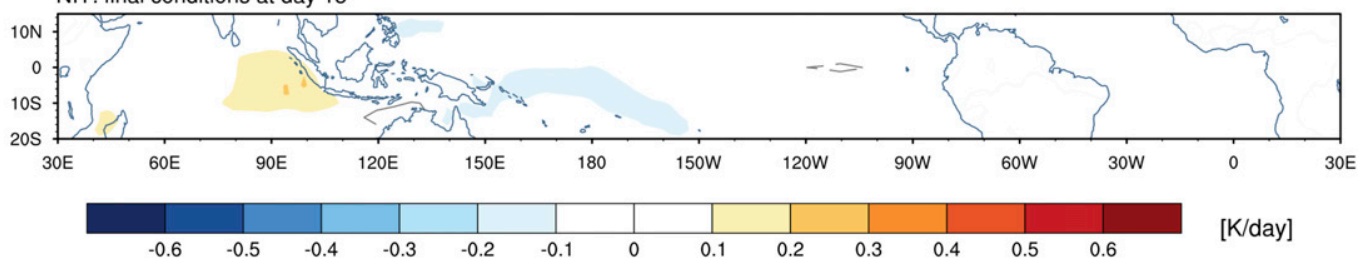

FIG. 12. Final conditions 15 days later for growth toward a PNA pattern for (a) 200-hPa (color shading) and 850$\mathrm{hPa}$ (contours) streamfunction and (b) tropical Q1 (color shading) and SST (contours) using modified optimal initial conditions for the full LIM. Two modifications to the initial conditions are shown, labeled NIE and NIT. For no initial extratropical conditions (NIE), initial conditions are modified by removing the extratropical 200- and 850hPa streamfunction initial conditions. For no initial tropical conditions (NIT), initial conditions are modified so that the tropical Q1 and SST initial conditions are removed. The 850-hPa streamfunction contours are every $1 \times$ $10^{6} \mathrm{~m}^{2} \mathrm{~s}^{-1}$, and SST contours are every $0.1 \mathrm{~K}$. All negative contours are dashed. Figures are scaled as in Fig. 2.

that the extratropical circulation is largely responsible for the PNA growth in the NIT case.

\section{Discussion and conclusions}

Linear inverse modeling is used to examine the optimal conditions leading to PNA pattern development and growth. In this diagnostic study, a full LIM is constructed from tropical SST, tropical Q1, and extratropical 200- and 850-hPa streamfunction anomalies. Results suggest that in the tropics, anomalous SPCZ and ENSO-related heating with oppositesigned MJO heating in the Indian Ocean optimally lead to PNA pattern growth. In the extratropics, optimal initial conditions are similar to the composite pattern in Franzke et al. (2011), including an anticyclonic anomaly over the northeast Pacific that retrogrades to become part of the PNA pattern. Decomposing the full LIM optimal initial conditions reveals that PNA growth is primarily due to the interference of strongly SST-coupled modes and internal atmospheric modes, with an opposite-signed behavior in the two subspaces for Q1 and streamfunction. Importantly, these two subspaces are not orthogonal, meaning their variances cannot be simply partitioned. The non-normality of the two subspaces is also evident in the power spectra of the PNA index, which shows that the full PNA variance 
a)
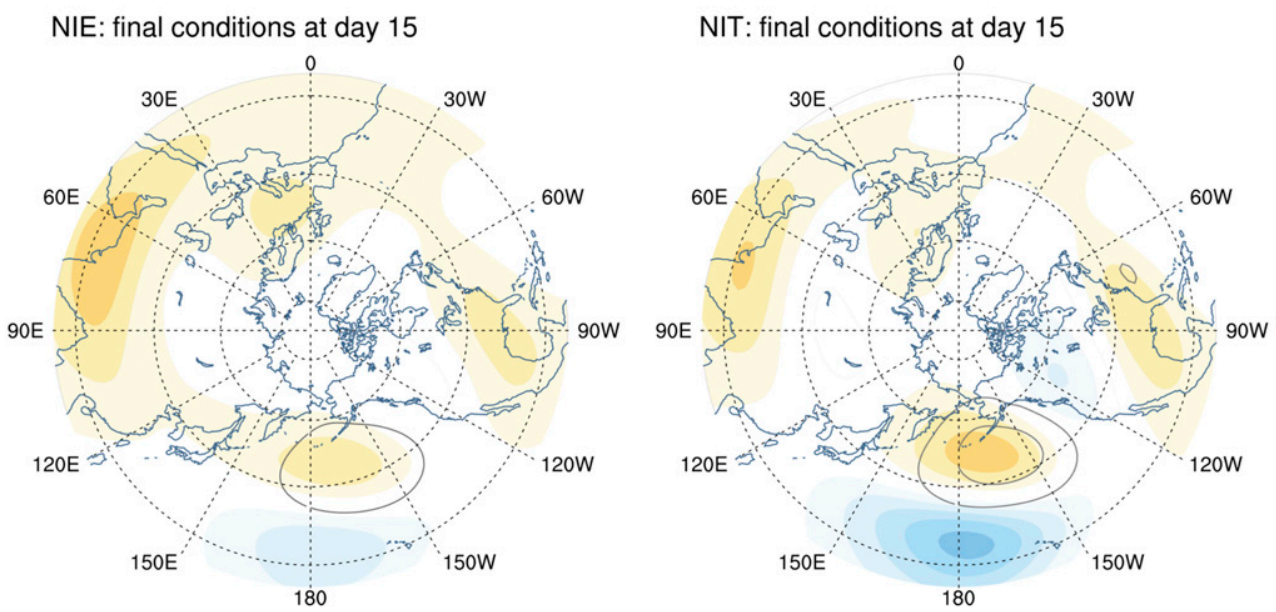

b)

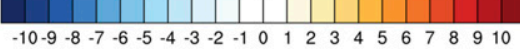

$\left[\times 10^{6} \mathrm{~m}^{2} / \mathrm{s}\right]$

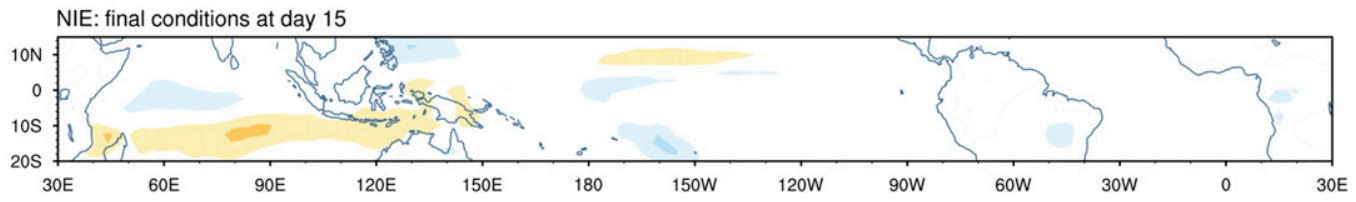

NIT: final conditions at day 15

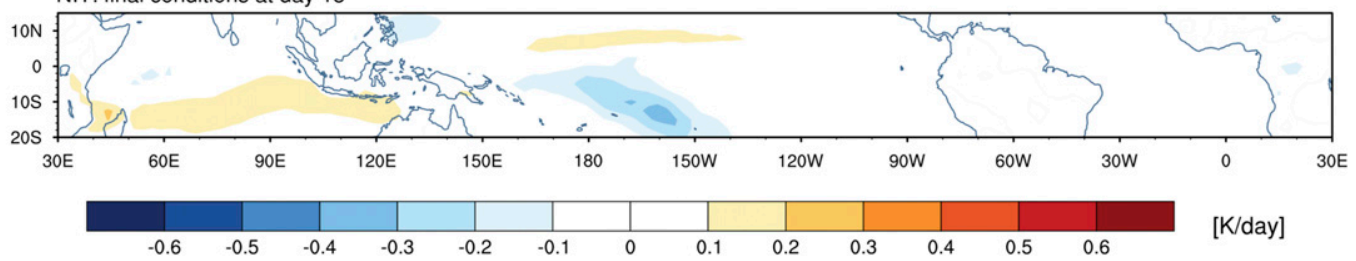

FIG. 13. As in Fig. 12, but for the internal atmospheric LIM. SST is excluded in the internal atmospheric LIM.

Figures are scaled as in Fig. 2.

is not simply the sum of variances from the coupled and internal atmospheric subspaces.

PNA growth independent of the interference between the coupled and internal atmospheric subspaces is examined through the development of an internal atmospheric LIM; that is, one that excludes SST-coupled dynamics by excluding the SST-coupled space. The evolution of the optimal Q1 for the internal atmospheric LIM, which as expected lacks an ENSO response, resembles the MJO with its characteristic eastward propagation, also captured in the full LIM. The Q1 optimal in both LIMs agrees with the findings of Mori and Watanabe (2008) and Henderson et al. (2016) that the MJO can trigger a PNA pattern through anomalous divergence over the Indian Ocean. In the extratropics, the optimal conditions leading to PNA growth are similar to those in the full LIM, with a northeast Pacific anticyclonic anomaly that retrogrades and becomes part of the PNA pattern. This indicates that the extratropical circulation anomalies that optimally lead to PNA growth are internal to the atmosphere and not related to strongly SST-coupled variability.

The relative importance of the extratropical and tropical initial conditions to PNA growth was examined by modifying the optimal initial conditions in the full and internal atmospheric LIMs. Modifying the full LIM initial conditions shows that both the tropical and extratropical fields are important for PNA pattern growth, with the tropical initial conditions forcing the strongest response. In the internal atmospheric LIM, both the tropical heating and the extratropical circulation are also important, with a slightly stronger contribution coming from the extratropical initial condition.

Previous studies have suggested the importance of non-normality for extratropical climate variability in general and the PNA in particular (e.g., Farrell 1988; Borges and Hartmann 1992; Borges and Sardeshmukh 
1995; Farrell and Ioannou 1996). A key result of our study is the importance of the non-normality between the SST-coupled and internal atmospheric subspaces for PNA growth. In fact, these results suggest that the PNA may be the consequence of a few different processes-including slowly evolving ENSO and more rapidly evolving MJO heat sources, and internal extratropical dynamics, potentially including those related to North Pacific blocking (see also Breeden et al. 2020)—which operate on different time scales to drive similar PNA-like upper tropospheric height anomalies. Rapid amplification of the PNA can then occur as these various processes drive component patterns evolving from destructive to constructive interference. Correspondingly, non-normality means that PNA variance is not so easily partitioned into contributions from different processes or phenomena. This could also be the case for other climate patterns beyond the PNA, as may be inferred from the appendix B figures, which are not specific to the PNA pattern. Our results therefore raise the more general point that caution should be taken in attribution studies, with consideration given to the non-normality of the coupled and internal atmospheric subspaces.

A novel contribution of this study is the development of an internal atmospheric LIM, which removes strongly SST-coupled modes from the dynamical operator, including ENSO. Note that this approach is fundamentally different than merely constructing the LIM with a state vector restricted to only atmospheric components, which would still retain much of the implicit effects of ENSO and other SST-coupled dynamics. The internal atmospheric LIM allows the study of optimal growth beyond ENSO, even for weather and climate patterns that are strongly influenced by ENSO. In this study, we have focused on optimal growth, defined using the maximum eigenvalue. Suboptimal PNA growth may also occur, as well as an unpredictable (noise-forced) PNA component, although this is beyond the scope of this study. Given the identification of the optimal initial conditions for PNA growth, future work includes further analysis to better understand the mechanisms behind these relationships. There is also ongoing work to better understand the impact of the extratropical circulation on MJO heating, as previously mentioned. Furthermore, the results presented here suggest that linear inverse modeling may be a useful tool for forecasting the PNA pattern, as has been done for other climate patterns in previous studies (e.g., Vimont 2012; Alexander et al. 2008).

Acknowledgments. This work was supported by the National Science Foundation under Grant AGS-1624831. Funding for DV was provided by the NOAA MAPP program, Grant OAR 4310097. MN was supported by NOAA/CPO/MAPP. We are grateful to two anonymous reviewers for their invaluable insight and to mathematician Josh Maglione at Universität Bielefeld for many useful linear algebra discussions. The statements, findings, conclusions, and recommendations do not necessarily reflect the views of NSF or NOAA.

Data availability statement. The ERA-Interim reanalysis 6-hourly data (Dee et al. 2011) can be accessed at https:// apps.ecmwf.int/datasets/data/interim-full-daily/levtype $=\mathrm{sfc} /$. The NOAA Optimum Interpolation Sea Surface Temperature (OISST) dataset (Reynolds et al. 2002) can be found at https://www.ncdc.noaa.gov/oisst/dataaccess. The NOAA/NCEP Climate Prediction Center (CPC) PNA index (CPC 2017) can be accessed at https:// www.cpc.ncep.noaa.gov/products/precip/CWlink/pna/ pna.shtml. The Wheeler and Hendon (2004) RMM MJO indices can be accessed at http://www.bom.gov.au/climate/ mjo/.

\section{APPENDIX A}

\section{Definition of "Growth"}

There are several ways of defining growth in the full and internal atmospheric LIMs. Using the 15-day optimal initial conditions for the PNA norm $\mathbf{p}_{1}$ growth, $\mu(\tau)$ from (4) can be quantified under the Euclidean (L2) norm (e.g., Vimont et al. 2014) or under the PNA norm for each LIM. PNA growth is plotted under the L2 norm (Fig. A1, left panel) and under the PNA norm (right panel) for the full (black curves) and internal atmospheric (red curves) LIMs, where "growth" is defined as $\mu(\tau)>1$. NIT (dashed curves) and NIE (dash-dotted curves) growth is also shown. However, note that the growth curves with no extratropical initial conditions (NIE; dash-dotted curves) could not be normalized to begin at 1 due to mathematical constraints.

For the full LIM, optimal PNA growth under the L2 norm is represented by two peaks, one near 20 days and the other near 87 days, with $\mu(\tau)>1$ for all lags shown. This longer time-scale growth is driven by the tropical ENSO anomalies that persist beyond the MJO heating (Fig. 3b), evidenced by the similar NIE growth curve (black dash-dotted curve). At time scales beyond 25 days, the final streamfunction anomalies are weakened relative to Fig. 2a (right panel) and more closely resemble the TNH pattern than the PNA pattern (not shown), highlighting the importance of non-ENSO anomalies in PNA growth. For the internal atmospheric LIM (solid red curve), growth maximizes within the first 10 days and there is decay $[\mu(\tau)<1]$ beyond 20 days, in agreement with the two-week PNA evolution time scale discussed in previous studies (e.g., Cash and Lee 2001; Franzke et al. 2011; Dai et al. 2017). For both the full and internal 

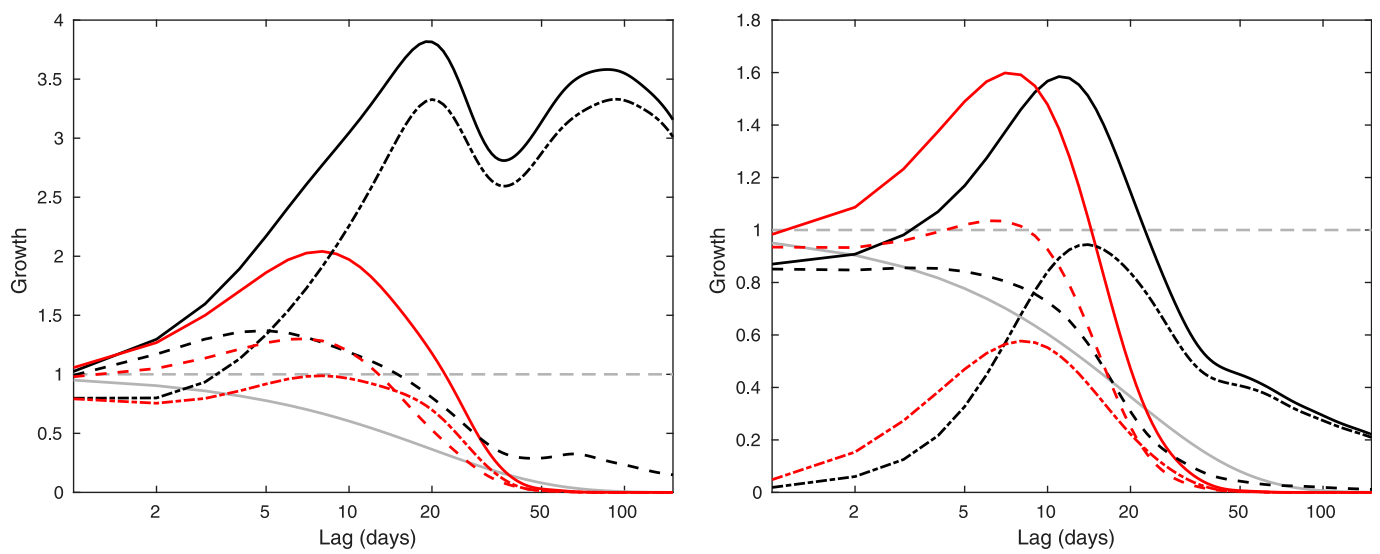

FIG. A1. Growth curves under (left) the L2 norm and (right) the PNA norm for the full (black curves) and internal atmospheric (red curves) LIMs. All optimal initial conditions are calculated using the PNA final norm in (4). NIE growth is shown by the dash-dotted curves and NIT growth curves are dashed. For reference, a gray dashed curve is shown for a growth value of 1 , and the gray solid curve is the expected decay via damped persistence of the PNA pattern.

atmospheric LIMs, comparisons to the NIT and NIE growth curves demonstrate that the greatest growth is measured when the full optimal initial condition is considered, in agreement with the discussion in section 6 .

One can further define "growth" by instead specifying PNA growth only under the PNA norm (Fig. A1, right panel). This method is less intuitive than the L2 norm given that the PNA itself only grows to a certain amplitude, but it is nevertheless useful in comparing the peak time scales of PNA "growth" between the different LIMs. For the full LIM (black solid curve), peak growth under the PNA norm occurs near 11 days, when the final pattern Pacific anomalies reach their maximum amplitude (e.g., day 10 shown in Fig. 3a, right panel). However, the PNA anomalies over North America more fully develop at slightly longer lags (Fig. 2a, right panel). Growth in the internal atmospheric LIM (solid red curve) peaks at 7 days, a slightly shorter time scale than the full LIM, suggesting that the internal atmospheric optimal initial conditions may provide PNA predictability at shorter time scales than the full LIM, as expected due to the absence of longer time scale SST-coupled variability. In addition, both the full and internal atmospheric LIM NIE growth curves show peaks at later lags than for NIT (dashed curves), indicative of the longer time needed for tropical heating to influence the PNA region.

\section{APPENDIX B}

\section{Testing the Filter}

Some evidence for the success of the filtering technique used in separating the MJO and ENSO is shown in
Newman et al. (2009). However, since our state vector variables are slightly different from theirs and because the filter is a critical component of the internal atmospheric LIM introduced in this study, in this section we provide some tests to ensure that ENSO is successfully filtered and that the internal atmospheric state vector retains MJO variability.

The total spatial variance of the full PCs (i.e., $\mathbf{x}$ ) and the filtered PCs (i.e., $\mathbf{x}^{\text {int }}$ and $\mathbf{x}^{\text {coup }}$ ) is provided for SST (Fig. B1a), Q1 (Fig. B1b), and 200-hPa streamfunction (Fig. B1c). In the full state vector, the spatial SST variance is greatest over the central and eastern tropical Pacific where ENSO occurs (Fig. B1a; top panel). This pattern is clear in the coupled SST state vector variance (bottom panel), and absent in $\mathbf{x}^{\text {int }}$ (middle panel), demonstrating that ENSO variance has been successfully removed. Similarly, Q1 variance in the coupled state vector (Fig. B1b, bottom panel) is primarily over the central Pacific, where ENSO heating is observed, while $\mathbf{x}^{\text {int }}$ retains most of the variance over the Indian Ocean and Maritime Continent (Fig. B1b, middle panel). Furthermore, most of the $200-\mathrm{hPa}$ streamfunction variance (Fig. B1c) is maintained by $\mathbf{x}^{\text {int }}$ (center panel), although some is lost likely due to the variance associated with ENSO teleconnections. When added, the variance of the internal atmospheric and coupled modes often exceeds that of the full LIM, indicating that these two subspaces are not orthogonal.

Power spectra are used as a check that $\mathbf{x}^{\text {coup }}$ contains the slower SST-coupled variability in $\mathbf{x}$, including ENSO, and $\mathbf{x}^{\text {int }}$ excludes ENSO and retains MJO variability. The statistics of the time series are obtained from a 20000-day run of (1), calculated by integrating (1) forward following the methodology of Penland and Matrosova (1994), also 
a)
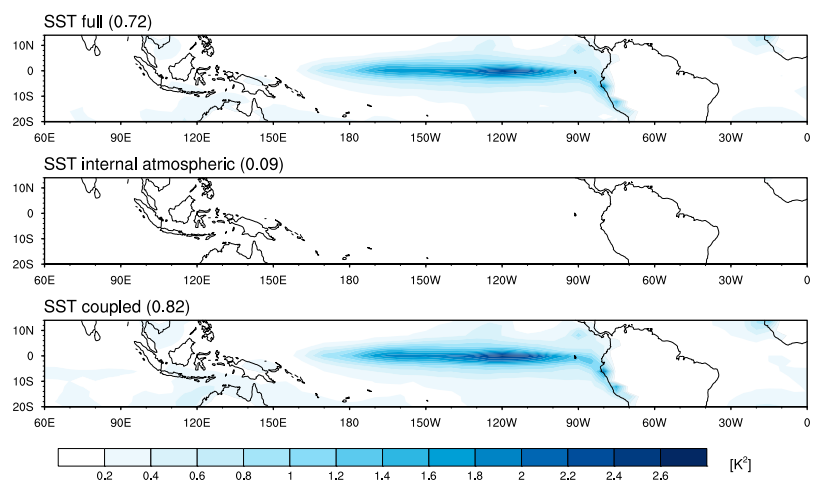

b)

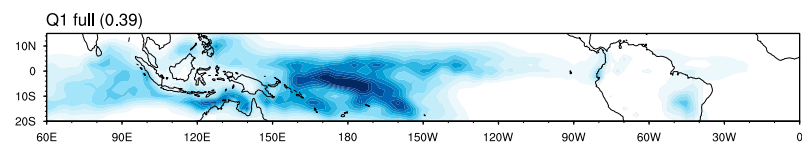

Q1 internal atmospheric (0.29)
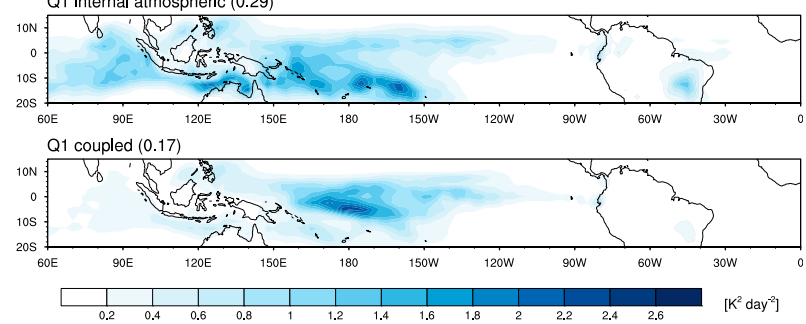

c)
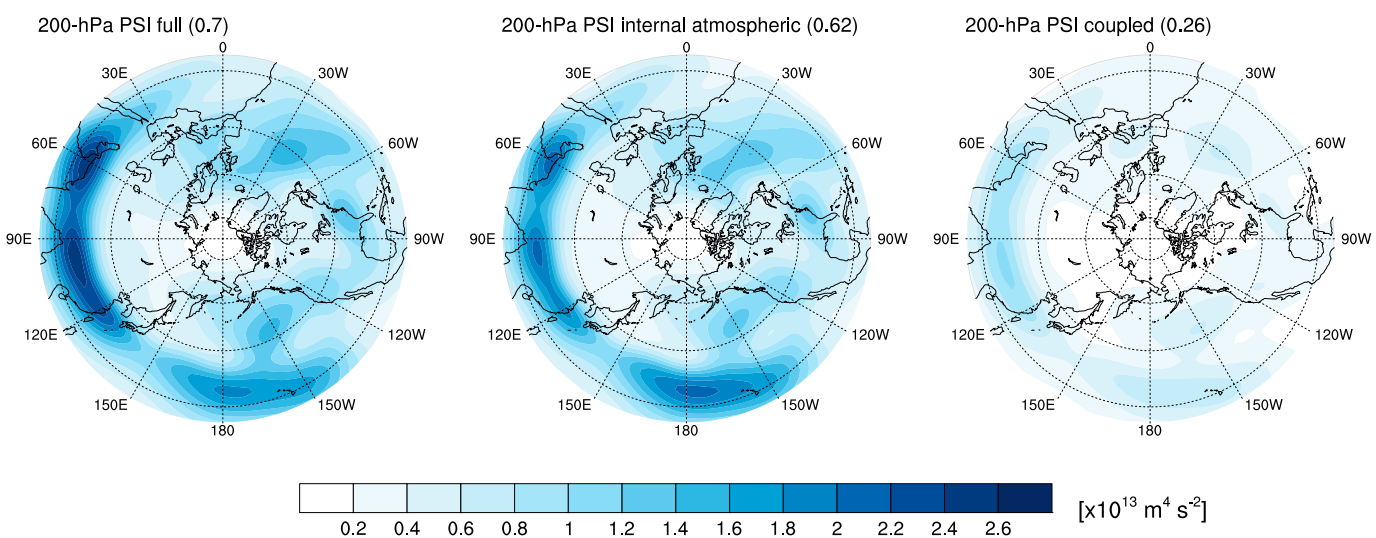

FIG. B1. Spatial variance in the full, internal atmospheric, and coupled state vector of (a) SST, (b) Q1, and (c) 200-hPa streamfunction. Above each panel is the total variance in parentheses.

used in Newman et al. (2009). The forward integration is calculated using the dynamical operator $\mathbf{L}$ and random Gaussian white noise forcing. The white noise forcing is obtained from the eigenvectors $\mathbf{q}$ and eigenvalues $\eta$ of the covariance matrix of the noise forcing $\mathbf{Q}$ that is derived as a residual of the fluctuation-dissipation relationship, $\mathbf{L} \mathbf{C}_{0}+\mathbf{C}_{0} \mathbf{L}^{\mathrm{T}}+\mathbf{Q}=0$. The forward integrated 20000-day time series, denoted as $\mathbf{X}$, is calculated as

$$
\mathbf{X}\left(t+\frac{\Delta}{2}\right)=\frac{[Y(t)+Y(t+\Delta)]}{2},
$$

where

$$
Y(t+\Delta)=Y(t)+\sum_{j} \mathbf{L}_{j} Y(t) \Delta+\sum_{i} \mathbf{q}_{i} \sqrt{\eta_{i} \Delta} \mathcal{R}_{i} .
$$

A set of normally distributed random numbers $\mathcal{R}$ is generated at each time step with unit variance, and $\Delta=$ $1 / 24$ days for a time step of $1 \mathrm{~h}$. The counters $j$ and $i$ range from 1 to 52 representing the $j$ th EOF from (6) and the $i$ th EOF of $\mathbf{Q}$. For more details on the forward integration, see
Penland and Matrosova (1994). Long run time series of the coupled and internal modes are calculated using (8), but using the long run $\mathbf{X}$ instead of the state vector $\mathbf{x}$.

The 20000-day time series are subdivided into 39 overlapping segments of 1000 days each and a Hann window is applied. The segments overlap by 500 days to account for the geometry of the Hann window. The observed spectra are then the average of the 39 spectral estimates. The power spectra of the three leading PCs of SST (top row), Q1 (middle row), and 200-hPa streamfunction (bottom row) are shown in Fig. B2. In blue and red are the spectra using the coupled and internal atmospheric modes, respectively. For reference, the power spectra calculated from the full (unfiltered) time series are provided in black. Again it is clear that the coupled and internal atmospheric subspaces are not orthogonal, with the spectra of the internal atmospheric and coupled modes often exceeding that of the full LIM. The coupled time series power spectra of the first two SST PCs include most of the low-frequency variance, including ENSO variability (Fig. B2, top row). This can be seen when 

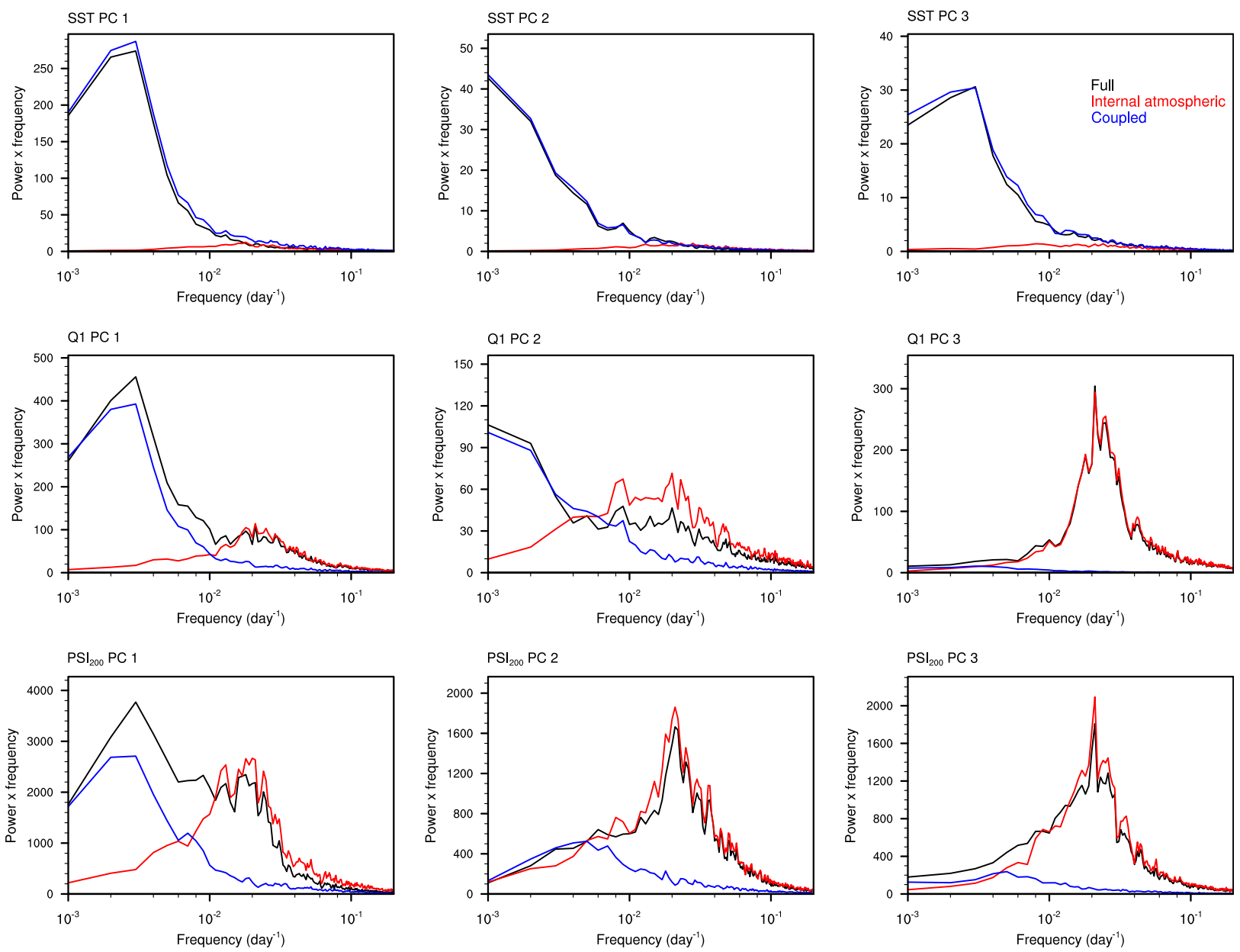

FIG. B2. Power spectra for the three leading PCs of (top) SST, (middle) Q1, and (bottom) 200-hPa streamfunction (PSI 200 ). Spectra calculated using the full PCs are shown in black. Also shown are the spectra of the PCs filtered by summing over the internal atmospheric modes of $\mathbf{L}$ (red curves) and by summing over the coupled modes of $\mathbf{L}$ (blue curves).

comparing the spectra of the coupled (blue curve) to that of the full (black curve) time series. This is also evident in the power spectra of the first two PCs of Q1 (middle row). For all Q1 power spectra, the intraseasonal and higherfrequency power is primarily in the internal atmospheric time series. Variability associated with the MJO is largely represented in Q1 PC 3 (middle row, right column) and PC 4 (not shown), both of which have most of the variance represented in the internal atmospheric time series.

Intraseasonal and higher-frequency variability is also primarily captured by the internal atmospheric $200-\mathrm{hPa}$ streamfunction time series (Fig. B2, bottom row), although some intraseasonal variability is reduced in the internal atmospheric PC 1 time series (red curve, bottom left panel). This can also be seen near 50 days in the power spectrum of Q1 PC 1 (middle row, left panel). The leading EOF of $200-\mathrm{hPa}$ streamfunction is a zonally symmetric pattern [not shown; see Fig. 1 of Frederiksen and Branstator (2005) for the 300-hPa leading pattern] that is very similar to the pattern that follows L2 growth in the LIM produced by Winkler et al. (2001). Their study demonstrated that this pattern is associated with a simultaneous ENSO and MJO, so that some of this variance may be reduced when decoupling from SST.

\section{REFERENCES}

Albers, J. R., and M. Newman, 2019: A priori identification of skillful extratropical subseasonal forecasts. Geophys. Res. Lett., 46, 12 527-12 536, https://doi.org/10.1029/2019GL085270.

Alexander, M. A., L. Matrosova, C. Penland, J. D. Scott, and P. Chang, 2008: Forecasting Pacific SSTs: Linear inverse model predictions of the PDO. J. Climate, 21, 385-402, https:// doi.org/10.1175/2007JCLI1849.1.

Borges, M. D., and D. L. Hartmann, 1992: Barotropic instability and optimal perturbations of observed nonzonal flows. J. Atmos. Sci., 49, 335-354, https://doi.org/10.1175/1520-0469(1992)049<0335: $\mathrm{BIAOPO}>2.0 . \mathrm{CO} ; 2$.

— , and P. D. Sardeshmukh, 1995: Barotropic Rossby wave dynamics of zonally varying upper-level flows during northern 
winter. J. Atmos. Sci., 52, 3779-3796, https://doi.org/10.1175/ 1520-0469(1995)052<3779:BRWDOZ>2.0.CO;2.

Boyd, J. P., 1983: The continuous spectrum of linear Couette flow with the beta effect. J. Atmos. Sci., 40, 2304-2308, https://doi.org/ 10.1175/1520-0469(1983)040<2304:TCSOLC $>2.0 . C O ; 2$.

Branstator, G., 1990: Low-frequency patterns induced by stationary waves. J. Atmos. Sci., 47, 629-649, https://doi.org/10.1175/ 1520-0469(1990)047<0629:LFPIBS > 2.0.CO;2.

_ 1992: The maintenance of low-frequency atmospheric anomalies. J. Atmos. Sci., 49, 1924-1946, https://doi.org/10.1175/15200469(1992)049<1924:TMOLFA > 2.0.CO;2.

Breeden, M. L., B. T. Hoover, M. Newman, and D. J. Vimont, 2020: Optimal North Pacific blocking precursors and their deterministic subseasonal evolution during boreal winter. Mon. Wea. Rev., 148, 739-761, https://doi.org/10.1175/MWR-D-19-0273.1.

Capotondi, A., and Coauthors, 2015: Understanding ENSO diversity. Bull. Amer. Meteor. Soc., 96, 921-938, https://doi.org/ 10.1175/BAMS-D-13-00117.1.

Cash, B. A., and S. Lee, 2001: Observed nonmodal growth of the Pacific-North American teleconnection pattern. J. Climate, 14, 1017-1028, https://doi.org/10.1175/1520-0442(2001)014<1017: ONGOTP $>2.0 . \mathrm{CO} ; 2$.

Casola, J. H., and J. M. Wallace, 2007: Identifying weather regimes in the wintertime 500-hPa geopotential height field for the Pacific-North American sector using a limited-contour clustering technique. J. Appl. Meteor. Climatol., 46, 1619-1630, https://doi.org/10.1175/JAM2564.1.

CPC, 2017: Normalized daily PNA indices. Accessed 25 May 2017, http://www.cpc.ncep.noaa.gov/products/precip/CWlink/daily_ ao_index/history/method.shtml\#reof.

Dai, Y., S. B. Feldstein, B. Tan, and S. Lee, 2017: Formation mechanisms of the Pacific-North American teleconnection with and without its canonical tropical convection pattern. J. Climate, 30, 3139-3155, https://doi.org/10.1175/JCLI-D-16-0411.1.

Dee, D. P., and Coauthors, 2011: The ERA-Interim reanalysis: Configuration and performance of the data assimilation system. Quart. J. Roy. Meteor. Soc., 137, 553-597, https://doi.org/ 10.1002/qj.828.

Dickson, R. R., and J. Namias, 1976: North American influences on the circulation and climate of the North Atlantic sector. Mon. Wea. Rev., 104, 1255-1265, https://doi.org/10.1175/1520-0493(1976)104<1255: NAIOTC $>2.0 . \mathrm{CO} ; 2$

Dole, R. M., and Coauthors, 2018: Advancing science and services during the 2015/16 El Niño. Bull. Amer. Meteor. Soc., 99, 9751001, https://doi.org/10.1175/BAMS-D-16-0219.1.

Farrell, B. F., 1982: The initial growth of disturbances in a baroclinic flow. J. Atmos. Sci., 39, 1663-1686, https://doi.org/ 10.1175/1520-0469(1982)039<1663:TIGODI > 2.0.CO;2.

_- 1988: Optimal excitation of neutral Rossby waves. J. Atmos. Sci., 45, 163-172, https://doi.org/10.1175/15200469(1988)045<0163:OEONRW $>2.0 . \mathrm{CO} ; 2$.

— Autonomous operators. J. Atmos. Sci., 53, 2025-2040, https:// doi.org/10.1175/1520-0469(1996)053<2025:GSTPIA > 2.0.CO;2.

$\longrightarrow$, and —, 1999: Optimal excitation of magnetic fields. Astrophys. J., 522, 1079-1087, https://doi.org/10.1086/307662.

Franzke, C., S. B. Feldstein, and S. Lee, 2011: Synoptic analysis of the Pacific-North American teleconnection pattern. Quart. J. Roy. Meteor. Soc., 137, 329-346, https://doi.org/10.1002/ qj. 768 .

Frederiksen, J. S., and G. Branstator, 2005: Seasonal variability of teleconnection patterns. J. Atmos. Sci., 62, 1346-1365, https:// doi.org/10.1175/JAS3405.1.
Henderson, D. S., C. D. Kummerow, and W. Berg, 2018: ENSO influence on TRMM tropical oceanic precipitation characteristics and rain rates. J. Climate, 31, 3979-3998, https://doi.org/10.1175/ JCLI-D-17-0276.1.

Henderson, S. A., E. D. Maloney, and E. A. Barnes, 2016: The influence of the Madden-Julian oscillation on Northern Hemisphere winter blocking. J. Climate, 29, 4597-4616, https://doi.org/10.1175/ JCLI-D-15-0502.1.

Hoerling, M. P., A. Kumar, and M. Zhong, 1997: El Niño, La Niña, and the nonlinearity of their teleconnections. J. Climate, $\mathbf{1 0}$, 1769-1786, https://doi.org/10.1175/1520-0442(1997)010<1769: ENOLNA $>2.0 . \mathrm{CO} ; 2$.

Horel, J. D., and J. M. Wallace, 1981: Planetary-scale atmospheric phenomena associated with the Southern Oscillation Mon. Wea. Rev., 109, 813-829, https://doi.org/10.1175/15200493(1981)109<0813:PSAPAW > 2.0.CO;2.

Jin, F., and B. J. Hoskins, 1995: The direct response to tropical heating in a baroclinic atmosphere. J. Atmos. Sci., 52, 307-319, https:// doi.org/10.1175/1520-0469(1995)052<0307:TDRTTH >2.0.CO;2.

Johnson, N. C., and S. B. Feldstein, 2010: The continuum of North Pacific sea level pressure patterns: Intraseasonal, interannual, and interdecadal variability. J. Climate, 23, 851-867, https:// doi.org/10.1175/2009JCLI3099.1.

Leathers, D. J., B. Yarnal, M. A. Palecki, D. J. Leathers, B. Yarnal, and M. A. Palecki, 1991: The Pacific/North American teleconnection pattern and United States climate. Part I: Regional temperature and precipitation associations. J. Climate, 4, 517-528, https://doi.org/ 10.1175/1520-0442(1991)004<0517:TPATPA >2.0.CO;2.

Lin, H., G. Brunet, and J. Derome, 2009: An observed connection between the North Atlantic oscillation and the MaddenJulian oscillation. J. Climate, 22, 364-380, https://doi.org/ 10.1175/2008JCLI2515.1.

Livezey, R. E., and K. C. Mo, 1987: Tropical-extratropical teleconnections during the Northern Hemisphere winter. Part II: Relationships between monthly mean Northern Hemisphere circulation patterns and proxies for tropical convection. Mon. Wea. Rev., 115, 3115-3132, https://doi.org/10.1175/ 1520-0493(1987)115<3115:TETDTN $>2.0$. CO; 2 .

Madden, R. A., and P. R. Julian, 1994: Observations of the 40-50-day tropical oscillation-A review. Mon. Wea. Rev., 122, 814 837, https://doi.org/10.1175/1520-0493(1994)122<0814: OOTDTO $>2.0 . \mathrm{CO} ; 2$

Martinez-Villalobos, C., and D. J. Vimont, 2016: The role of the mean state in meridional mode structure and growth. J. Climate, 29, 3907-3921, https://doi.org/10.1175/JCLI-D-15-0542.1.

Matthews, A. J., B. J. Hoskins, and M. Masutani, 2004: The global response to tropical heating in the Madden-Julian oscillation during the northern winter. Quart. J. Roy. Meteor. Soc., 130, 1991-2011, https://doi.org/10.1256/qj.02.123.

Mo, K., and M. Ghil, 1988: Cluster analysis of multiple planetary flow regimes. J. Geophys. Res., 93, 10 927-10 952, https:// doi.org/10.1029/JD093iD09p10927.

Moore, A. M., and R. Kleeman, 1999: The nonnormal nature of El Niño and intraseasonal variability. J. Climate, 12, 2965-2982, https:// doi.org/10.1175/1520-0442(1999)012<2965:TNNOEN>2.0.CO;2.

Mori, M., and M. Watanabe, 2008: The growth and triggering mechanisms of the PNA: A MJO-PNA coherence. J. Meteor. Soc. Japan, 86, 213-236, https://doi.org/10.2151/jmsj.86.213.

Newman, M., 2007: Interannual to decadal predictability of tropical and North Pacific sea surface temperatures. J. Climate, 20, 2333-2356, https://doi.org/10.1175/JCLI4165.1. 
2013: An empirical benchmark for decadal forecasts of global surface temperature anomalies. J. Climate, 26, 5260-5269, https://doi.org/10.1175/JCLI-D-12-00590.1.

— and P. D. Sardeshmukh, 1998: The impact of the annual cycle on the North Pacific/North American response to remote lowfrequency forcing. J. Atmos. Sci., 55, 1336-1353, https://doi.org/ 10.1175/1520-0469(1998)055<1336:TIOTAC >2.0.CO;2.

$\longrightarrow$, and - 2008: Tropical and stratospheric influences on extratropical short-term climate variability. J. Climate, 21, 43264347, https://doi.org/10.1175/2008JCLI2118.1.

,,-- C. R. Winkler, and J. S. Whitaker, 2003: A study of subseasonal predictability. Mon. Wea. Rev., 131, 1715-1732, https://doi.org/10.1175//2558.1.

,-- , and C. Penland, 2009: How important is air-sea coupling in ENSO and MJO evolution? J. Climate, 22, 2958-2977, https://doi.org/10.1175/2008JCLI2659.1.

— , and Coauthors, 2016: The Pacific decadal oscillation, revisited. J. Climate, 29, 4399-4427, https://doi.org/10.1175/ JCLI-D-15-0508.1.

Penland, C., and L. Matrosova, 1994: A balance condition for stochastic numerical models with application to the El NiñoSouthern Oscillation. J. Climate, 7, 1352-1372, https://doi.org/ 10.1175/1520-0442(1994)007<1352:ABCFSN >2.0.CO;2.

— , and P. D. Sardeshmukh, 1995: The optimal growth of tropical sea surface temperature anomalies. J. Climate, 8, 1999-2024, https:// doi.org/10.1175/1520-0442(1995)008<1999:TOGOTS > 2.0.CO;2.

_ , and L. Matrosova, 1998: Prediction of tropical Atlantic sea surface temperatures using linear inverse modeling. J. Climate, 11, 483-496, https://doi.org/10.1175/1520-0442(1998)011<0483:POTASS > 2.0.CO;2.

_ in tropical sea surface temperatures using a nonnormal filter. J. Climate, 19, 5796-5815, https://doi.org/10.1175/JCLI3951.1.

Reynolds, R. W., N. A. Rayner, T. M. Smith, D. C. Stokes, and W. Wang, 2002: An improved in situ and satellite SST analysis for climate. J. Climate, 15, 1609-1625, https://doi.org/10.1175/ 1520-0442(2002)015<1609:AIISAS>2.0.CO;2.

Riddle, E. E., M. B. Stoner, N. C. Johnson, M. L. L. Heureux, D. C. Collins, and S. B. Feldstein, 2012: The impact of the MJO on clusters of wintertime circulation anomalies over the North American region. Climate Dyn., 40,1749-1766, https://doi.org/ 10.1007/S00382-012-1493-Y.

Sardeshmukh, P. D., M. Newman, and M. D. Borges, 1997: Free barotropic Rossby wave dynamics of the wintertime lowfrequency flow. J. Atmos. Sci., 54, 5-23, https://doi.org/ 10.1175/1520-0469(1997)054<0005:FBRWDO >2.0.CO;2.
Simmons, A. J., J. M. Wallace, and G. W. Branstator, 1983: Barotropic wave propagation and instability, and atmospheric teleconnection patterns. J. Atmos. Sci., 40,1363-1392, https://doi.org/10.1175/15200469(1983)040<1363:BWPAIA $>2.0$. CO;2

Strang, G., 2006: Linear Algebra and Its Applications. 4th ed. Thomson, Brooks/Cole, 487 pp

Straus, D. M., and J. Shukla, 2000: Distinguishing between the SST-forced variability and internal variability in mid latitudes: Analysis of observations and GCM simulations. Quart. J. Roy. Meteor. Soc., 126, 2323-2350, https://doi.org/10.1002/qj.49712656716.

— , and - 2002: Does ENSO force the PNA? J. Climate, 15, 2340-2358, https://doi.org/10.1175/1520-0442(2002)015<2340: DEFTP $>2.0 . \mathrm{CO} ; 2$.

Tziperman, E., L. Zanna, and C. Penland, 2008: Nonnormal thermohaline circulation dynamics in a coupled ocean-atmosphere GCM. J. Phys. Oceanogr., 38, 588-604, https://doi.org/10.1175/ 2007JPO3769.1.

Vimont, D. J., 2012: Analysis of the Atlantic meridional mode using linear inverse modeling: Seasonality and regional influences. J. Climate, 25, 1194-1212, https://doi.org/10.1175/JCLI-D-11-00012.1.

- M. A. Alexander, and M. Newman, 2014: Optimal growth of central and East Pacific ENSO events. Geophys. Res. Lett., 41, 4027-4034, https://doi.org/10.1002/2014GL059997.

von Storch, H., G. Burger, R. Schnur, and J.-S. von Storch, 1995: Principal oscillation patterns: A review. J. Climate, 8, 377-400, https://doi.org/ 10.1175/1520-0442(1995)008<0377:POPAR > 2.0.CO;2.

Waliser, D., and Coauthors, 2009: MJO simulation diagnostics. J. Climate, 22, 3006-3030, https://doi.org/10.1175/2008JCLI2731.1.

Wallace, J. M., and D. S. Gutzler, 1981: Teleconnections in the geopotential height field during the Northern Hemisphere winter. Mon. Wea. Rev., 109, 784-812, https://doi.org/10.1175/ 1520-0493(1981)109<0784:TITGHF $>2.0$.CO;2.

Wheeler, M. C., and H. H. Hendon, 2004: An all-season real-time multivariate MJO index: Development of an index for monitoring and prediction. Mon. Wea. Rev., 132, 1917-1932, https://doi.org/10.1175/ 1520-0493(2004)132<1917:AARMMI>2.0.CO;2.

Winkler, C. R., M. Newman, and P. D. Sardeshmukh, 2001: A linear model of wintertime low-frequency variability. Part I: Formulation and forecast skill. J. Climate, 14, 4474-4494, https://doi.org/10.1175/ 1520-0442(2001)014<4474:ALMOWL>2.0.CO;2.

Yanai, M., S. Esbensen, and J.-H. Chu, 1973: Determination of bulk properties of tropical cloud clusters from large-scale heat and moisture budgets. J. Atmos. Sci., 30, 611-627, https://doi.org/ 10.1175/1520-0469(1973)030<0611:DOBPOT>2.0.CO;2.

Zhang, C., 2005: Madden-Julian Oscillation. Rev. Geophys., 43 , RG2003, https://doi.org/10.1029/2004RG000158. 
